\title{
24. CHEMICAL COMPOSITION OF SEDIMENTS
}

Yu. A. Bogdanov, A.P. Lisitzin, V.N. Lukashin, V.V. Gordeev, I.B. Zverinskaya, B.B. Zeljdina, A.D. Kuzinov, and V.N. Zhivago, P.P. Shirshov Institute of Oceanology, Academy of Sciences of the USSR, Moscow

The chemical composition of oceanic bottom sediments depends upon many factors, including geographic and vertical zonality of the sedimentary processes. The distribution of such "abiogenic" components as $\mathrm{TiO}_{2}$ and $\mathrm{MnO}$ is determined by the nature of mechanical differentiation. Abiogenic pelagic marine sediments form a continuous lithologic facies series, the end members of which are terrigenous and metal-bearing oozes. The state of the sediment in relation to this series also reflects its "degree of pelaginicity." We assume that the metal-bearing oozes are primarily formed by local hydrothermal exhalations or weathering of basalts. The chemical elements and compounds which occur in minor amounts in the sediments and do not form independent minerals pose a complicated problem. Whether they can be used as indicators of depositional environments has not yet been resolved.

In order to analyze the chemical composition of bottom sediments, we used several methods which enabled us to get information on a sufficiently wide spectrum of chemical elements and duplicate the determinations by various methods. We determined the content of the following elements and compounds and these data are presented in Tables 1-9. Techniques are described in the Initial Reports of the Deep Sea Drilling Project, Volume 6.

1) Spectral rational silicate analysis- $\mathrm{SiO}_{2}, \mathrm{Al}_{2} \mathrm{O}_{3}$, $\mathrm{CaO}, \mathrm{MgO}, \mathrm{TiO}_{2}, \mathrm{Fe}_{2} \mathrm{O}_{3}, \mathrm{MnO}$ (Table 1);

2) Flame photometry- $\mathrm{K}_{2} \mathrm{O}, \mathrm{Na}_{2} \mathrm{O}, \mathrm{Li}, \mathrm{Rb}, \mathrm{Cs}$ (Table 2);

3) Spectral-Ga, Cr, Ni, V, Zr, Y, Co;

4) Atomic absorption- $\mathrm{Ni}, \mathrm{Zn}, \mathrm{Cu}$;

5) Neutron activation- $\mathrm{Hf}, \mathrm{Rb}, \mathrm{Ta}, \mathrm{Sm}, \mathrm{Fe}, \mathrm{Se}, \mathrm{Co}$, $\mathrm{La}, \mathrm{Eu}, \mathrm{Sb}, \mathrm{Ce}, \mathrm{Th}, \mathrm{Cr}, \mathrm{Cs}, \mathrm{Ba}$ (Table 3);

6) "Wet" chemistry- $\mathrm{C}_{\text {orr }}, \mathrm{CO}_{2}, \mathrm{SiO}_{2}$ amorph, $\mathrm{Fe}_{2} \mathrm{O}_{3}$, $\mathrm{TiO}_{2}, \mathrm{MnO}, \mathrm{CaO}, \mathrm{MgO}, \mathrm{P}_{2} \mathrm{O}_{5}$ (Tables 4-8);

7) Determinations of $\mathrm{C}_{\text {org }}$ and $\mathrm{C}_{\text {carb }}$ by means of “LECO” analyzer (Table 9).

\section{DISTRIBUTION OF MAJOR ELEMENTS IN BOTTOM SEDIMENTS}

\section{$\mathrm{CaCO}_{3}, \mathrm{CaO}, \mathrm{MgO}$}

Most of the samples obtained during Leg 35 were noncalcareous because this part of the Southeast Pacific Basin lies in a zone where production of biogenic carbonate is sharply curtailed, and the carbonate compensation depth (CCD) lies considerably shallower than the water depth of the sites. However, the Paleocene sediments of Site 323 contained interbedded layers of coccolith-rich sediment composed of $28 \%-29 \% \mathrm{CaCO}_{3}$. Typi-

\footnotetext{
TTranslated from Russian.
}

cal deep-water pelagic oozes, which in some places resemble the metal-bearing oozes, were deposited above and below the zone of coccolith interbeds. At Site 323 sediment accumulated at depths close to the CCD, and therefore, small fluctuations of the CCD sufficed for the formation of discrete calcareous interlayers. Some interlayers of coccolith-containing sediments of Oligocene age were also encountered at Site 325 .

$\mathrm{CaO}$ and $\mathrm{MgO}$, except in the calcareous horizons, are abiogenic components of the sediments. The fluctuations in their concentration in space and time are determined by changes in the mineral composition. The average $\mathrm{CaO}$ and $\mathrm{MgO}$ concentrations and the $\mathrm{CaO} / \mathrm{MgO}$ ratios are given in Table 10 .

The sediments of the Bellingshausen Sea and adjacent areas differ from the typical pelagic sediments analyzed by El-Wakeel and Riley (1961). We recognize two areas based on variations of $\mathrm{CaO}$ and $\mathrm{MgO}$ content in the sediments: the western area (Sites 323 and 324) and the eastern area (Sites 322 and 325). In the sediments of the eastern area the $\mathrm{CaO}$ and $\mathrm{MgO}$ content approximates the average crustal composition of the silicic and terrigenous rocks, whereas in the sediments of the western area, the $\mathrm{CaO}$ content is similar to that of pelagic oozes, and $\mathrm{MgO}$ is somewhat lower. The values of the $\mathrm{CaO} / \mathrm{MgO}$ ratio in coarse sediments are considerably higher than in the thin pelitic oozes.

\section{$\mathrm{SiO}_{2}$}

The principal silica-containing components of the bottom sediments are abiogenic silicates, quartz, and biogenic remains of diatoms, radiolarians, silicoflagellates, and sponges. There is silicification in discrete interlayers as a result of diagenetic alterations.

To determine the content of biogenic silica we used the sodium carbonate extraction method, which generally yields somewhat lower results but permitted us to ascertain the trend of the changes in the accumulation of biogenic silica (Figure 1).

In the Upper Cretaceous and Paleogene sediments the content of amorphous silica rarely exceeded $2 \%$. At the onset of the Neogene, the accumulation of biogenic opal greatly increased and sediments of Neogene and Pleistocene age contained $5 \%-10 \%$ and in some places even greater amounts of amorphous silica even though it comprises less than $2 \%$ in individual interlayers. This is caused by dilution of the biogenic opal with terrigenous material, whose supply was rather sporadic.

The content of the amorphous silica in bottom sediments can also be determined by the amount of accumulated $\mathrm{SiO}_{2} / \mathrm{Al}_{2} \mathrm{O}_{3}$. Despite the fact that the area is characterized by a high production of biogenic siliceous material, we rarely encountered siliceous sediments because of their extensive dilution by terrigenous 
TABLE 1

Content of Major Elements in Sediments and Sedimentary Rocks, in \% of Dry Weight (Spectral Silicate Analysis)

\begin{tabular}{|c|c|c|c|c|c|c|c|c|}
\hline $\begin{array}{c}\text { Sample } \\
\text { (Interval in } \mathrm{cm} \text { ) }\end{array}$ & $\begin{array}{l}\text { Depth } \\
(\mathrm{m})\end{array}$ & $\mathrm{SiO}_{2}$ & $\mathrm{TiO}_{2}$ & $\mathrm{Al}_{2} \mathrm{O}_{3}$ & $\mathrm{Fe}_{2} \mathrm{O}_{3}$ & $\mathrm{MnO}$ & $\mathrm{MgO}$ & $\mathrm{CaO}$ \\
\hline \multicolumn{9}{|l|}{ Site 322} \\
\hline $\begin{array}{l}1-2,26-30 \\
1-8,70-76 \\
3-1,18-20 \\
4-1,65-67 \\
4-2,57-60 \\
5-1,81-85 \\
6-1,30-33 \\
9-2,143-146 \\
10-1,101-105 \\
10-2,43-46 \\
11-2,45-49 \\
11-3,24-32 \\
11-4,17-24 \\
11-6,109-115 \\
13-2\end{array}$ & $\begin{array}{r}80 \\
81 \\
295 \\
353 \\
354 \\
391 \\
438 \\
469 \\
486 \\
487 \\
506 \\
507 \\
508 \\
512 \\
537\end{array}$ & $\begin{array}{l}64 \\
66 \\
50 \\
54 \\
64 \\
56 \\
56 \\
57 \\
66 \\
62 \\
66 \\
68 \\
63 \\
58 \\
50\end{array}$ & $\begin{array}{l}0.66 \\
1.0 \\
1.4 \\
1.2 \\
1.0 \\
1.3 \\
1.2 \\
1.0 \\
0.72 \\
0.92 \\
1.1 \\
1.6 \\
0.84 \\
1.4 \\
2.3\end{array}$ & $\begin{array}{l}11 \\
12 \\
14 \\
13 \\
13 \\
15 \\
14 \\
15 \\
13 \\
12 \\
12 \\
14 \\
12 \\
20 \\
15\end{array}$ & $\begin{array}{l}3.5 \\
5.7 \\
5.8 \\
6.7 \\
7.4 \\
6.2 \\
7.4 \\
5.1 \\
4.5 \\
4.6 \\
4.9 \\
5.6 \\
4.1 \\
11 \\
12\end{array}$ & $\begin{array}{l}0.058 \\
0.10 \\
0.11 \\
0.11 \\
0.10 \\
0.14 \\
0.58 \\
0.084 \\
0.061 \\
0.072 \\
0.081 \\
0.090 \\
0.090 \\
>0.60 \\
0.20\end{array}$ & $\begin{array}{l}1.0 \\
2.6 \\
4.6 \\
4.0 \\
2.8 \\
2.1 \\
2.3 \\
2.0 \\
1.8 \\
1.6 \\
3.0 \\
2.6 \\
1.8 \\
3.6 \\
6.6\end{array}$ & $\begin{array}{l}2.7 \\
2.3 \\
2.9 \\
2.6 \\
3.3 \\
3.4 \\
1.7 \\
4.8 \\
5.1 \\
3.7 \\
2.9 \\
2.9 \\
2.9 \\
1.4 \\
8.1\end{array}$ \\
\hline \multicolumn{9}{|l|}{ Site 323} \\
\hline $\begin{array}{l}1-1,50-60 \\
1-1,140-150 \\
1-2,34-43 \\
1-3,78-88 \\
1-4,118-127 \\
3-1,88-94 \\
3-2,14-20 \\
3-2,61-70 \\
4-2, \\
7-2,105-110 \\
7-3,18-24 \\
10-1,113-122 \\
10-2,75-85 \\
10-3,106-114 \\
11-1,22-32 \\
11-2,137-148 \\
13-5,106-115 \\
13-6,145-150 \\
14-2,1-8 \\
14-2,128-135 \\
15-1,52-60 \\
15-2,91-100 \\
15-3,29-36 \\
15-4,54-63 \\
15-5,89-98 \\
15-6,19-26 \\
16-1,57-62 \\
16-3,32-37 \\
16-4,83-92 \\
18-2,80-86 \\
18-3,130-139 \\
18-4,115-124 \\
18-5,65-73\end{array}$ & $\begin{array}{r}76 \\
77 \\
79 \\
80 \\
81 \\
257 \\
258 \\
258 \\
315 \\
363 \\
364 \\
504 \\
505 \\
507 \\
551 \\
553 \\
624 \\
625 \\
637 \\
638 \\
656 \\
657 \\
658 \\
660 \\
661 \\
663 \\
665 \\
668 \\
669 \\
695 \\
697 \\
699 \\
700\end{array}$ & $\begin{array}{l}56 \\
57 \\
56 \\
52 \\
54 \\
55 \\
58 \\
56 \\
55 \\
52 \\
58 \\
64 \\
60 \\
60 \\
55 \\
51 \\
47 \\
51 \\
52 \\
48 \\
45 \\
50 \\
47 \\
45 \\
46 \\
33 \\
34 \\
46 \\
61 \\
46 \\
45 \\
64 \\
57\end{array}$ & $\begin{array}{l}0.32 \\
0.55 \\
0.65 \\
0.59 \\
0.58 \\
0.62 \\
0.40 \\
0.67 \\
0.32 \\
0.58 \\
0.60 \\
0.61 \\
0.55 \\
0.60 \\
0.63 \\
0.64 \\
0.59 \\
0.57 \\
0.51 \\
0.63 \\
0.60 \\
0.67 \\
0.51 \\
0.58 \\
0.55 \\
0.39 \\
0.49 \\
0.46 \\
0.63 \\
0.52 \\
0.55 \\
0.47 \\
0.48\end{array}$ & $\begin{array}{c}8.0 \\
9.6 \\
12 \\
11 \\
10 \\
12 \\
9.6 \\
10 \\
9.8 \\
8.5 \\
10 \\
21 \\
10 \\
10 \\
14 \\
11 \\
10 \\
7.9 \\
8.4 \\
15 \\
10 \\
15 \\
14 \\
8.4 \\
9.8 \\
10 \\
7.8 \\
9.6 \\
12 \\
11 \\
10 \\
12 \\
12\end{array}$ & $\begin{array}{l}1.9 \\
5.1 \\
7.1 \\
6.6 \\
5.9 \\
5.0 \\
2.9 \\
7.3 \\
2.9 \\
7.0 \\
6.6 \\
6.4 \\
6.8 \\
6.4 \\
6.2 \\
5.6 \\
5.5 \\
4.4 \\
4.5 \\
9.8 \\
8.5 \\
9.8 \\
8.3 \\
7.4 \\
7.7 \\
6.2 \\
5.1 \\
6.5 \\
7.9 \\
5.7 \\
5.6 \\
7.4 \\
8.9\end{array}$ & $\begin{array}{l}0.16 \\
0.21 \\
0.13 \\
0.094 \\
0.074 \\
0.080 \\
0.060 \\
0.052 \\
0.075 \\
0.15 \\
0.14 \\
0.070 \\
0.093 \\
0.094 \\
0.10 \\
0.094 \\
0.13 \\
0.12 \\
0.10 \\
0.53 \\
0.53 \\
0.73 \\
0.52 \\
0.57 \\
0.60 \\
0.54 \\
0.65 \\
0.22 \\
0.53 \\
0.079 \\
0.070 \\
\sim 1.0 \\
\sim 1.0\end{array}$ & $\begin{array}{l}0.7 \\
1.6 \\
2.4 \\
2.2 \\
2.0 \\
2.0 \\
1.0 \\
2.4 \\
1.0 \\
2.1 \\
2.4 \\
2.1 \\
2.3 \\
2.2 \\
2.2 \\
1.9 \\
2.1 \\
1.8 \\
1.4 \\
2.0 \\
2.3 \\
2.2 \\
2.2 \\
2.0 \\
1.7 \\
1.3 \\
1.7 \\
2.2 \\
1.4 \\
1.4\end{array}$ & $\begin{array}{l}1.4 \\
1.2 \\
1.0 \\
1.3 \\
1.0 \\
1.4 \\
1.8 \\
1.4 \\
1.8 \\
1.4 \\
1.6 \\
1.8 \\
1.2 \\
1.2 \\
1.0 \\
1.2 \\
1.0 \\
0.90 \\
1.0 \\
1.6 \\
1.3 \\
1.1 \\
1.0 \\
1.1 \\
1.0 \\
12 \\
10 \\
1.4 \\
1.8 \\
1.0 \\
1.2 \\
1.3 \\
1.3\end{array}$ \\
\hline \multicolumn{9}{|l|}{ Site 324} \\
\hline $\begin{array}{l}1-2,126-131 \\
1-3,124-132 \\
1-4,125-131 \\
1-6,31-38 \\
2-1,83-92 \\
2-2,61-70 \\
2-3,52-61 \\
2-4,27-36 \\
2-5,35-45 \\
2-6,95-104 \\
3-1,137-146 \\
3-2,136-145 \\
3-3,67-77 \\
3-4,47-57\end{array}$ & $\begin{array}{l}11 \\
13 \\
15 \\
17 \\
48 \\
49 \\
50 \\
52 \\
53 \\
55 \\
77 \\
79 \\
80 \\
81\end{array}$ & $\begin{array}{l}54 \\
60 \\
60 \\
57 \\
54 \\
54 \\
58 \\
54 \\
60 \\
54 \\
58 \\
56 \\
52 \\
65\end{array}$ & $\begin{array}{l}0.35 \\
0.69 \\
0.75 \\
0.64 \\
0.77 \\
0.92 \\
0.75 \\
0.63 \\
0.63 \\
0.82 \\
1.2 \\
1.1 \\
1.0 \\
1.6\end{array}$ & $\begin{array}{c}12 \\
8.4 \\
\sim 10 \\
8.4 \\
9.4 \\
11 \\
11 \\
9.3 \\
9.0 \\
8.5 \\
13 \\
16 \\
16 \\
23\end{array}$ & $\begin{array}{l}4.9 \\
5.5 \\
5.5 \\
4.7 \\
5.6 \\
5.9 \\
6.4 \\
5.1 \\
4.8 \\
5.2 \\
5.5 \\
5.7 \\
6.1 \\
7.6\end{array}$ & $\begin{array}{l}0.15 \\
0.14 \\
0.72 \\
0.11 \\
0.075 \\
0.095 \\
0.066 \\
0.056 \\
0.054 \\
0.11 \\
0.087 \\
0.085 \\
0.095 \\
0.11\end{array}$ & $\begin{array}{l}1.4 \\
1.4 \\
1.7 \\
1.8 \\
1.4 \\
1.7 \\
1.8 \\
1.6 \\
1.8 \\
2.1 \\
2.3\end{array}$ & $\begin{array}{l}1.4 \\
1.3 \\
1.7 \\
1.2 \\
0.96 \\
0.98 \\
1.1 \\
1.2 \\
1.2 \\
1.1 \\
1.2 \\
1.3 \\
1.5 \\
1.0\end{array}$ \\
\hline
\end{tabular}


TABLE 1 - Continued

\begin{tabular}{|c|c|c|c|c|c|c|c|c|}
\hline $\begin{array}{c}\text { Sample } \\
\text { (Interval in cm) }\end{array}$ & $\begin{array}{l}\text { Depth } \\
\text { (m) }\end{array}$ & $\mathrm{SiO}_{2}$ & $\mathrm{TiO}_{2}$ & $\mathrm{Al}_{2} \mathrm{O}_{3}$ & $\mathrm{Fe}_{2} \mathrm{O}_{3}$ & $\mathrm{MnO}$ & $\mathrm{MgO}$ & $\mathrm{CaO}$ \\
\hline \multicolumn{9}{|c|}{ Site 324 - Continued } \\
\hline $\begin{array}{l}3-5,99-110 \\
3-6,138-148 \\
4-2,113-123 \\
4-3,30-40 \\
4-4,54-64 \\
5-2,66-83 \\
5-3,25-35 \\
6-2,19-30 \\
7-1,69-78 \\
7-3,110-121 \\
7-6,119-129\end{array}$ & $\begin{array}{r}83 \\
85 \\
107 \\
108 \\
109 \\
135 \\
136 \\
144 \\
153 \\
156 \\
161\end{array}$ & $\begin{array}{l}62 \\
67 \\
64 \\
60 \\
66 \\
62 \\
68 \\
60 \\
62 \\
58 \\
63\end{array}$ & $\begin{array}{l}1.2 \\
1.1 \\
1.3 \\
1.2 \\
1.4 \\
1.8 \\
1.8 \\
1.3 \\
1.9 \\
1.3 \\
1.8\end{array}$ & $\begin{array}{l}16 \\
16 \\
14 \\
15 \\
18 \\
18 \\
20 \\
16 \\
21 \\
18 \\
20\end{array}$ & $\begin{array}{l}6.9 \\
6.8 \\
6.1 \\
6.0 \\
6.6 \\
6.9 \\
6.7 \\
6.2 \\
7.6 \\
6.5 \\
7.7\end{array}$ & $\begin{array}{l}0.094 \\
0.063 \\
0.094 \\
0.10 \\
0.15 \\
0.12 \\
0.11 \\
0.085 \\
0.11 \\
0.75 \\
0.10\end{array}$ & $\begin{array}{l}1.9 \\
1.8 \\
1.6 \\
1.6 \\
2.0 \\
2.7 \\
2.2 \\
1.8 \\
2.6 \\
1.6 \\
2.2\end{array}$ & $\begin{array}{l}1.0 \\
1.5 \\
1.1 \\
1.4 \\
2.8 \\
1.2 \\
1.2 \\
1.4 \\
1.2 \\
1.2 \\
1.2\end{array}$ \\
\hline \multicolumn{9}{|l|}{ Site 325} \\
\hline $\begin{array}{l}1-1,49-59 \\
1-1,133-144 \\
1-2,26-36 \\
1-4,81-92 \\
2-2,39-49 \\
3-2,11-24 \\
3-3,76-87 \\
3-4,26-36 \\
4-2,90 \\
4-3,20-26 \\
4-3,130 \\
4, C C \\
5-1,55-57 \\
8-1,45-49 \\
8-2,119-128 \\
8-3,27-35 \\
9-3,90-98\end{array}$ & $\begin{array}{r}34 \\
35 \\
36 \\
39 \\
168 \\
178 \\
180 \\
182 \\
293 \\
294 \\
295 \\
295 \\
404 \\
613 \\
615 \\
616 \\
645\end{array}$ & $\begin{array}{l}50 \\
48 \\
47 \\
56 \\
50 \\
56 \\
63 \\
62 \\
64 \\
60 \\
58 \\
60 \\
57 \\
54 \\
58 \\
57 \\
53\end{array}$ & $\begin{array}{l}0.94 \\
0.75 \\
0.83 \\
0.80 \\
0.58 \\
0.59 \\
0.55 \\
0.72 \\
0.69 \\
0.57 \\
0.79 \\
0.62 \\
0.77 \\
0.61 \\
0.56 \\
0.64 \\
0.81\end{array}$ & $\begin{array}{c}9.1 \\
7.7 \\
8.4 \\
7.9 \\
6.1 \\
6.6 \\
6.2 \\
7.4 \\
11 \\
6.8 \\
9.0 \\
12 \\
9.3 \\
7.9 \\
8.2 \\
8.2 \\
10\end{array}$ & $\begin{array}{l}6.9 \\
5.2 \\
6.1 \\
5.7 \\
4.5 \\
4.4 \\
4.0 \\
4.6 \\
5.1 \\
5.0 \\
5.3 \\
5.1 \\
6.1 \\
5.1 \\
5.2 \\
4.9 \\
6.4\end{array}$ & $\begin{array}{l}0.098 \\
0.10 \\
0.11 \\
0.098 \\
0.10 \\
0.085 \\
0.073 \\
0.098 \\
0.14 \\
0.095 \\
0.11 \\
0.17 \\
0.11 \\
0.098 \\
0.14 \\
0.10 \\
0.10\end{array}$ & $\begin{array}{l}2.1 \\
2.2 \\
2.5 \\
2.2 \\
2.0 \\
2.2 \\
1.4 \\
1.9 \\
2.2 \\
1.6 \\
2.0 \\
2.6 \\
2.4 \\
2.0 \\
2.2 \\
2.3 \\
2.4\end{array}$ & $\begin{array}{l}2.1 \\
2.8 \\
2.5 \\
2.3 \\
2.1 \\
2.0 \\
2.1 \\
2.3 \\
2.6 \\
2.5 \\
2.6 \\
2.6 \\
2.1 \\
2.1 \\
4.6 \\
2.0 \\
2.2\end{array}$ \\
\hline
\end{tabular}

sediments. More often, the siliceous sediments occurred as discrete layers. The amounts of $\mathrm{SiO}_{2} / \mathrm{Al}_{2} \mathrm{O}_{3}$ and amorphous silica in the cores that we studied indicated that accumulation of biogenic opal began near the boundary between Paleogene and Neogene time.

\section{$\mathrm{TiO}_{2}$}

Titantium, as well as aluminum, is considered by many investigators to be an indicator of sediments from a terrigenous source. The sediments and sedimentary rocks recovered during DSDP Leg 35 contain considerably more titanium than do pelagic oozes. A direct correlation between amounts of $\mathrm{Al}$ and $\mathrm{Ti}$ in the sediments could not be established. The $\mathrm{Al}_{2} \mathrm{O}_{3} / \mathrm{TiO}_{2}$ ratio varied from 9 to 34 ; in the coarser grained sediments it was sometimes greater and in other cases it remained similar to the values established for clayey oozes. An increase of silicate detritus in the silt and sand fraction results in an increase of the aluminum content and an increase of the $\mathrm{Al}_{2} \mathrm{O}_{3} / \mathrm{TiO}_{2}$ ratio. In these cases, when quartz is the predominant constituent of the coarse fractions and the content of silicates is relatively small, the value of $\mathrm{Al}_{2} \mathrm{O}_{3} / \mathrm{TiO}_{2}$ remains closely equivalent to the value established for pelitic oozes.

\section{$\mathrm{Fe}_{2} \mathrm{O}_{3}$ and $\mathrm{MnO}$}

Iron and manganese occur in sediments in various forms; the two most common forms are in crystalline lattices of detrital and clayey minerals, and in the free state-most frequently in the form of hydroxides which form independent colloidal, aggregates, or as occlusions on mineral particles.
The content of iron and manganese in the sediments decreased with increased grain size and varied in the pelitic oozes. At Sites 322 and 323, there are interlayers in Paleogene sediments which are considerably enriched in iron and manganese.

The $\mathrm{Fe}_{2} \mathrm{O}_{3} / \mathrm{MnO}$ ratio varies from 9 to 140 . High values characterize the terrigenous sediments, but the values are markedly less in metal-bearing oozes (Figure 2).

\section{$\mathbf{C}_{\text {org }}$ and $\mathbf{P}$}

Because of the distinctive distribution of the organic carbon, phosphorous, and values of $\mathrm{C}_{\text {org }} / \mathrm{P}$, the contact between the Paleogene and Neogene is clearly discernible. The amount of organic carbon in the Upper Cretaceous and Paleogene sediments was relatively small but increased during the Neogene. The concentration of phosphorous, in contrast, was high in the lower part of the sedimentary sequence, gradually increased toward the upper layer of the Paleogene sediments, and in the Neogene abruptly decreased. The lower part of the sedimentary sequence is characterized by $\mathrm{C}_{\text {org }} / \mathrm{P}$ values of less than 1 (and which in some places are less than 0.1), whereas in the Neogene and Pleistocene sediments, values are usually considerably higher than 1 .

From evaluation of the lithologic composition of the sediments and distribution of the chemical indicators, we believe that during the Upper Cretaceous and Paleogene, the depositional environment in the southeast Pacific Basin was similar to that presently found in the ocean under the arid climatic zones $\left(10^{\circ}\right.$ - 
TABLE 2

Content of $\mathrm{Na}_{2} \mathrm{O}, \mathrm{K}_{2} \mathrm{O}, \mathrm{Li}, \mathrm{Rb}$, and $\mathrm{Cs}$ in the Sediments of Sedimentary Rocks in \% of Dry Weight (Flame Photometry)

\begin{tabular}{|c|c|c|c|c|c|c|}
\hline $\begin{array}{c}\text { Sample } \\
\text { (Interval in } \mathrm{cm} \text { ) }\end{array}$ & $\begin{array}{l}\text { Depth } \\
\text { (m) }\end{array}$ & $\mathrm{Na}_{2} \mathrm{O}$ & $\mathrm{K}_{2} \mathrm{O}$ & $\mathrm{Li}$ & $\mathrm{Rb}$ & Cs \\
\hline \multicolumn{7}{|l|}{ Site 322} \\
\hline $\begin{array}{l}1-2,26-30 \\
1-3,70-76 \\
3-1,18-20 \\
4-1,65-67 \\
4-2,57-60 \\
5-1,81-85 \\
6-1,30-33 \\
9-2,143-146 \\
10-1,101-105 \\
10-2,43-46 \\
11-2,45-49 \\
11-3,24-32 \\
11-4,17-24 \\
11-6,109-115 \\
13-2\end{array}$ & $\begin{array}{r}80 \\
81 \\
295 \\
353 \\
354 \\
391 \\
438 \\
469 \\
486 \\
487 \\
506 \\
507 \\
508 \\
512 \\
537\end{array}$ & $\begin{array}{l}4.00 \\
3.87 \\
3.46 \\
3.22 \\
3.22 \\
3.00 \\
2.72 \\
3.65 \\
3.57 \\
3.57 \\
3.15 \\
3.50 \\
3.57 \\
1.72 \\
2.45\end{array}$ & $\begin{array}{l}1.89 \\
2.16 \\
2.34 \\
2.22 \\
1.95 \\
2.43 \\
2.70 \\
1.53 \\
1.53 \\
1.41 \\
1.50 \\
1.74 \\
1.81 \\
3.75 \\
1.14\end{array}$ & $\begin{array}{l}0.0012 \\
0.0022 \\
0.0036 \\
0.0026 \\
0.0019 \\
0.0027 \\
0.003 \\
0.0009 \\
0.0011 \\
0.0012 \\
0.0009 \\
0.0012 \\
0.00125 \\
0.00375 \\
0.0010\end{array}$ & $\begin{array}{l}0.007 \\
0.009 \\
0.0105 \\
0.0082 \\
0.0137 \\
0.0087 \\
0.019 \\
0.0055 \\
0.005 \\
0.005 \\
0.0042 \\
0.0042 \\
0.0045 \\
0.01775 \\
0.00175\end{array}$ & 0.0001 \\
\hline \multicolumn{7}{|l|}{ Site 323} \\
\hline $\begin{array}{l}1-1,50-60 \\
1-1,140-150 \\
1-2,34-43 \\
1-3,78-88 \\
1-4,118-127 \\
3-1,88-94 \\
3-2,14-20 \\
3-2,61-70 \\
4-2, \\
7-2,105-110 \\
7-3,18-24 \\
10-1,113-122 \\
10-2,75-85 \\
10-3,106-114 \\
11-1,22-32 \\
11-2,137-148 \\
13-5,106-115 \\
13-6,145-150 \\
14-2,1-8 \\
14-2,128-135 \\
15-1,52-60 \\
15-2,91-100 \\
15-3,29-36 \\
15-4,54-63 \\
15-5,89-98 \\
15-6,19-26 \\
16-1,57-62 \\
16-3,32-37 \\
16-4,83-92 \\
18-2,80-86 \\
18-3,130-139 \\
18-4,115-124 \\
18-5,65-73\end{array}$ & $\begin{array}{r}76 \\
77 \\
79 \\
80 \\
81 \\
257 \\
258 \\
258 \\
315 \\
363 \\
364 \\
504 \\
505 \\
507 \\
551 \\
553 \\
624 \\
625 \\
637 \\
638 \\
656 \\
657 \\
658 \\
660 \\
661 \\
663 \\
665 \\
668 \\
669 \\
695 \\
697 \\
699 \\
700\end{array}$ & $\begin{array}{l}4.05 \\
4.39 \\
3.95 \\
2.97 \\
4.76 \\
4.35 \\
4.05 \\
4.23 \\
4.35 \\
3.88 \\
3.88 \\
3.71 \\
3.65 \\
3.71 \\
3.51 \\
3.37 \\
2.77 \\
2.43 \\
2.56 \\
2.56 \\
2.43 \\
2.43 \\
2.26 \\
2.43 \\
2.90 \\
1.03 \\
1.37 \\
2.42 \\
2.70 \\
2.25 \\
2.65 \\
2.57 \\
2.42\end{array}$ & $\begin{array}{l}2.37 \\
2.94 \\
3.27 \\
2.70 \\
2.70 \\
2.88 \\
2.16 \\
2.88 \\
3.03 \\
2.10 \\
2.55 \\
2.61 \\
3.03 \\
2.34 \\
3.69 \\
3.09 \\
3.36 \\
3.27 \\
3.42 \\
2.52 \\
2.82 \\
3.15 \\
3.01 \\
3.24 \\
3.42 \\
2.34 \\
2.34 \\
2.70 \\
2.91 \\
3.15 \\
3.24 \\
2.34 \\
2.34\end{array}$ & $\begin{array}{l}0.0011 \\
0.0025 \\
0.0033 \\
0.0027 \\
0.0029 \\
0.0027 \\
0.0019 \\
0.0046 \\
0.0017 \\
0.0033 \\
0.0033 \\
0.0031 \\
0.0031 \\
0.0029 \\
0.0031 \\
0.0027 \\
0.0046 \\
0.0029 \\
0.0036 \\
0.0063 \\
0.007 \\
0.0066 \\
0.007 \\
0.0072 \\
0.0068 \\
0.0044 \\
0.0030 \\
0.0028 \\
0.0025 \\
0.0025 \\
0.0026 \\
0.0028 \\
0.0026\end{array}$ & $\begin{array}{l}0.0115 \\
0.018 \\
0.0197 \\
0.0132 \\
0.0152 \\
0.0145 \\
0.012 \\
0.018 \\
0.014 \\
0.015 \\
0.0145 \\
0.0127 \\
0.0170 \\
0.0172 \\
0.020 \\
0.0172 \\
0.0175 \\
0.016 \\
0.0172 \\
0.009 \\
0.0092 \\
0.0115 \\
0.0115 \\
0.0115 \\
0.012 \\
0.0077 \\
0.0077 \\
0.0087 \\
0.0102 \\
0.009 \\
0.016 \\
0.011 \\
0.0092\end{array}$ & $\begin{array}{l}0.0003 \\
0.0018 \\
0.0018 \\
0.0007 \\
0.0014 \\
0.0007 \\
0.0003 \\
0.0010 \\
0.0003 \\
0.0010 \\
0.0007 \\
0.0007 \\
0.0007 \\
0.0007 \\
0.0010 \\
0.0010 \\
0.0007 \\
0.0003 \\
0.0007 \\
0.0003 \\
\\
0.0003 \\
0.0003 \\
0.0001\end{array}$ \\
\hline \multicolumn{7}{|l|}{ Site 324} \\
\hline $\begin{array}{l}1-2,126-131 \\
1-3,124-132 \\
1-4,125-131 \\
1-6,31-38 \\
2-1,83-92 \\
2-2,61-70 \\
2-3,52-61 \\
2-4,27-36 \\
2-5,35-45 \\
2-6,95-104 \\
3-1,137-146 \\
3-2,136-145 \\
3-3,67-77\end{array}$ & $\begin{array}{l}11 \\
13 \\
15 \\
17 \\
48 \\
49 \\
50 \\
52 \\
53 \\
55 \\
77 \\
79 \\
80\end{array}$ & $\begin{array}{l}3.42 \\
2.95 \\
2.75 \\
2.85 \\
2.40 \\
2.40 \\
2.50 \\
2.67 \\
2.57 \\
2.57 \\
2.57 \\
2.50 \\
2.40\end{array}$ & $\begin{array}{l}3.00 \\
3.06 \\
3.72 \\
3.12 \\
3.96 \\
3.81 \\
3.37 \\
3.30 \\
3.51 \\
3.48 \\
3.57 \\
3.48 \\
3.42\end{array}$ & $\begin{array}{l}0.0031 \\
0.003 \\
0.0033 \\
0.0026 \\
0.0036 \\
0.004 \\
0.0041 \\
0.0033 \\
0.0037 \\
0.0035 \\
0.003 \\
0.0035 \\
0.0043\end{array}$ & $\begin{array}{l}0.0159 \\
0.01525 \\
0.019 \\
0.0145 \\
0.0225 \\
0.023 \\
0.0185 \\
0.0192 \\
0.0205 \\
0.0192 \\
0.019 \\
0.0182 \\
0.0189\end{array}$ & $\begin{array}{l}0.0004 \\
0.00055 \\
0.0003 \\
0.0004 \\
0.00055 \\
0.00055 \\
0.00015\end{array}$ \\
\hline
\end{tabular}


TABLE 2 - Continued

\begin{tabular}{|c|c|c|c|c|c|c|}
\hline $\begin{array}{c}\text { Sample } \\
\text { (Interval in } \mathrm{cm} \text { ) }\end{array}$ & $\begin{array}{l}\text { Depth } \\
\text { (m) }\end{array}$ & $\mathrm{Na}_{2} \mathrm{O}$ & $\mathrm{K}_{2} \mathrm{O}$ & $\mathrm{Li}$ & $\mathrm{Rb}$ & Cs \\
\hline \multicolumn{7}{|c|}{ Site 324 - Continued } \\
\hline $\begin{array}{l}3-4,47-57 \\
3-5,99-110 \\
3-6,138-148 \\
4-2,113-123 \\
4-3,30-40 \\
4-4,56-64 \\
5-2,66-83 \\
5-3,25-35 \\
6-2,19-30 \\
7-1,69-78 \\
7-3,110-121 \\
7-6,119-129 \\
8-3,61-71\end{array}$ & $\begin{array}{r}81 \\
83 \\
85 \\
107 \\
108 \\
109 \\
135 \\
136 \\
144 \\
153 \\
156 \\
161 \\
174\end{array}$ & $\begin{array}{l}2.40 \\
2.50 \\
2.75 \\
2.50 \\
2.57 \\
2.15 \\
2.57 \\
2.32 \\
2.15 \\
2.10 \\
2.15 \\
2.10 \\
2.22\end{array}$ & $\begin{array}{l}3.57 \\
3.81 \\
3.00 \\
3.00 \\
3.42 \\
2.88 \\
3.00 \\
3.15 \\
3.75 \\
3.48 \\
3.48 \\
3.57 \\
4.20\end{array}$ & $\begin{array}{l}0.00415 \\
0.0039 \\
0.0033 \\
0.0038 \\
0.00365 \\
0.0033 \\
0.0033 \\
0.0033 \\
0.00365 \\
0.00385 \\
0.00365 \\
0.00385 \\
0.00355\end{array}$ & $\begin{array}{l}0.01975 \\
0.02025 \\
0.0155 \\
0.018 \\
0.020 \\
0.0155 \\
0.01775 \\
0.0205 \\
0.02325 \\
0.02225 \\
0.02325 \\
0.0235 \\
0.023\end{array}$ & $\begin{array}{l}0.0004 \\
0.0004 \\
0.0004 \\
0.0006 \\
0.0005 \\
0.0003 \\
0.0004 \\
0.0003 \\
0.0005 \\
0.0004 \\
0.0005 \\
0.0005 \\
0.0004\end{array}$ \\
\hline \multicolumn{7}{|l|}{ Site 325} \\
\hline $\begin{array}{l}1-1,49-59 \\
1-1,133-144 \\
1-2,26-36 \\
1-4,81-92 \\
2-2,39-49 \\
3-2,11-24 \\
3-3,76-87 \\
3-4,26-36 \\
4-2,90 \\
4-3,20-26 \\
4-3,130 \\
4, C C \\
5-1,55-57 \\
8-1,45-49 \\
8-2,119-128 \\
8-3,27-35 \\
9-3,90-98\end{array}$ & $\begin{array}{r}34 \\
35 \\
36 \\
39 \\
168 \\
178 \\
180 \\
182 \\
293 \\
294 \\
295 \\
295 \\
404 \\
613 \\
615 \\
616 \\
645 \\
\end{array}$ & $\begin{array}{l}2.87 \\
3.00 \\
3.05 \\
3.15 \\
2.92 \\
2.72 \\
3.02 \\
3.10 \\
3.17 \\
3.10 \\
3.37 \\
3.17 \\
3.02 \\
2.70 \\
2.40 \\
2.62 \\
2.82\end{array}$ & $\begin{array}{l}2.58 \\
2.16 \\
2.28 \\
2.07 \\
1.98 \\
1.77 \\
1.56 \\
1.84 \\
1.92 \\
1.68 \\
1.80 \\
1.87 \\
2.175 \\
1.84 \\
1.95 \\
2.22 \\
2.07\end{array}$ & $\begin{array}{l}0.0031 \\
0.0032 \\
0.0032 \\
0.0026 \\
0.0017 \\
0.0025 \\
0.00165 \\
0.00175 \\
0.0016 \\
0.0017 \\
0.0016 \\
0.0016 \\
0.00295 \\
0.0026 \\
0.0025 \\
0.00275 \\
0.00275\end{array}$ & $\begin{array}{l}0.009 \\
0.0085 \\
0.009 \\
0.0067 \\
0.0055 \\
0.0082 \\
0.0065 \\
0.007 \\
0.007 \\
0.0065 \\
0.0065 \\
0.007 \\
0.0095 \\
0.00775 \\
0.010 \\
0.01025 \\
0.010\end{array}$ & 0.0001 \\
\hline
\end{tabular}

$30^{\circ} \mathrm{N}, 0^{\circ}-30^{\circ} \mathrm{S}$ ) and that conditions changed abruptly at the boundary between the Paleogene and the Neogene.

\section{Trace Elements}

The distribution of trace elements in the DSDP Leg 35 cores shows that their accumulation during various geological periods was rather sporadic. In the Upper Cretaceous and Paleogene at Sites 322 and 323, we encountered deposits resembling the metal-bearing sediments of the Bauer depression. At Site 322 these deposits, which overlie the basalts, were approximately 11 meters thick, whereas at Site 323 they were over 40 meters thick. The relatively low concentrations of $\mathrm{Si}, \mathrm{Al}$, and $\mathrm{Ti}$, the high content of $\mathrm{Fe}$ and, especially, of $\mathrm{Mn}$, as well as the presence of interbedded coccolith ooze indicate a minor terrigenous contribution to the sediment which was deposited under arid latitude conditions. High concentrations of $\mathrm{Ni}, \mathrm{Co}, \mathrm{Sc}$, rare earth elements, $\mathrm{V}$, and $\mathrm{Ba}$ were found in Cretaceous and Paleogene sediments which contained only minor amounts of hydrolysates $(\mathrm{Ga}, \mathrm{Zr}, \mathrm{Hf}, \mathrm{Th}), \mathrm{Cr}$, and alkaline metal. The concentrations of $\mathrm{Ti}, \mathrm{Rb}, \mathrm{Cs}$, and $\mathrm{Ba}$ in the sediments were usually lower than their average crustal concentrations. The increased concentration of barium in the sediments is due to the formation of barite, as a result of hydrothermal interactions between $\mathrm{Ba}^{+2}$ and sulfates contained in seawater. At Site 322 we had only one sample of metal-bearing sediment, and consequently we analyzed the entire sequence from the cores of Site
323. Profiles of selected trace element, iron, and manganese concentrations at Site 323 are presented in Figure 3.

The basalts were leached upon contact with cold water which greatly influenced the chemical composition of the sediments forming above the basalts. Iron and manganese precipitated from the leached basalts in the form of hydrous oxides simultaneously with the other elements adsorbed by them. Further accumulation of bottom sediments occurred under conditions of a minimal influx of terrigenous material similar to the sedimentary process presently occurring in the ocean under arid zones. Toward the end of the Paleogene the hydrothermal activity increased. Hydrothermal solutions entered the lower part of the sedimentary strata and percolated upward through the sediment interstices. In some cases, authigenic barite was precipitated where mixing of hydrothermal and interstitial waters occurred. This was noted only in the lower horizons of the unit. The upward diffusion of the hydrothermal solutions caused a gradual change in $p \mathrm{H}$ and $\mathrm{Eh}$ and thus changes in the physical and chemical environment.

In other cases, the hydrothermal solutions reached the sea-floor surface, oxidized, and formed a distinctive sediment which was enriched with hydrous ferric oxides, $\mathrm{Fe}, \mathrm{Mn}$, and trace elements. This material could have been carried by bottom currents to considerable distances. 
TABLE 3

Content of Trace Elements in Sediments in $10^{-4} \%$ of Dry Weight

\begin{tabular}{|c|c|c|c|c|c|c|c|c|c|c|c|c|c|c|c|c|c|c|c|c|c|c|c|c|c|c|}
\hline \multirow{2}{*}{$\begin{array}{c}\text { Sample } \\
\text { (Interval in } \mathrm{cm} \text { ) }\end{array}$} & \multirow{2}{*}{$\begin{array}{c}\text { Depth } \\
(\mathrm{m})\end{array}$} & \multicolumn{7}{|c|}{ Spectral Analysis } & & $\begin{array}{l}\text { Itomic } \\
\text { sorptior }\end{array}$ & & & & & & & & Neutron & Activati & in Analy & & & & & & \\
\hline & & Ga & $\mathrm{Cr}$ & $\mathrm{Ni}$ & $\mathrm{Y}$ & $\mathrm{Fr}_{\mathrm{r}}$ & $\mathrm{Y}$ & Co & $\mathrm{Ni}$ & $\mathrm{Zn}$ & $\mathrm{Cu}$ & $\mathrm{Hf}$ & $\mathrm{Rb}$ & $\mathrm{Ta}$ & $\mathrm{Sm}$ & $\mathrm{Fe} \%$ & $\mathrm{Sc}$ & Co & $\mathrm{La}$ & Eu & $\mathrm{Sb}$ & $\mathrm{Ce}$ & Th & $\mathrm{Cr}_{\mathrm{r}}$ & Cs & Ba \\
\hline Site 322 & & & & & & & & & & & & & & & & & & & & & & & & & & \\
\hline $\begin{array}{l}1-2,28-30 \\
1-3,70-76\end{array}$ & $\begin{array}{l}80 \\
81\end{array}$ & & $\begin{array}{l}20 \\
47\end{array}$ & 3 & $\begin{array}{l}38 \\
58\end{array}$ & $\begin{array}{l}52 \\
58\end{array}$ & $\begin{array}{l}12 \\
12\end{array}$ & 12 & $\begin{array}{l}12 \\
16\end{array}$ & $\begin{array}{l}48 \\
83\end{array}$ & $\begin{array}{l}18 \\
70\end{array}$ & $\begin{array}{l}2.95 \\
3.35\end{array}$ & $\begin{array}{l}38.6 \\
570\end{array}$ & 0.6 & $\begin{array}{l}3.9 \\
4.15\end{array}$ & 2.8 & 14.1 & $\begin{array}{r}8.7 \\
13.8\end{array}$ & 25.2 & 1.3 & 0.66 & $\begin{array}{l}30.7 \\
53.1\end{array}$ & 5.53 & 18.8 & 1.69 & 470 \\
\hline $3-1,18-20$ & $\begin{array}{r}81 \\
295\end{array}$ & $\begin{array}{l}20 \\
16\end{array}$ & $\begin{array}{l}47 \\
28\end{array}$ & 16 & $\begin{array}{l}58 \\
80\end{array}$ & $\begin{array}{l}58 \\
55\end{array}$ & $\begin{array}{l}12 \\
14\end{array}$ & $\begin{array}{l}13 \\
17\end{array}$ & $\begin{array}{l}16 \\
20\end{array}$ & $\begin{array}{r}83 \\
104\end{array}$ & 60 & $\begin{array}{l}3.35 \\
3.4\end{array}$ & $\begin{array}{l}5.0 \\
65.0\end{array}$ & $\begin{array}{l}1.1 \\
0.96\end{array}$ & $\begin{array}{l}4.15 \\
4.15\end{array}$ & $\begin{array}{l}4.3 \\
6.3\end{array}$ & $\begin{array}{l}22.3 \\
29.2\end{array}$ & $\begin{array}{l}13.8 \\
20.2\end{array}$ & $\begin{array}{l}26.8 \\
36.7\end{array}$ & $\begin{array}{l}1.5 \\
1.6\end{array}$ & $\begin{array}{l}0.94 \\
0.01\end{array}$ & $\begin{array}{l}53.1 \\
56.0\end{array}$ & $\begin{array}{r}8.1 \\
11.0\end{array}$ & $\begin{array}{l}33.5 \\
46.5\end{array}$ & $\begin{array}{l}3.1 \\
5.7\end{array}$ & $\begin{array}{l}430 \\
560\end{array}$ \\
\hline $4-1,65-67$ & 353 & 19 & 32 & 11 & 84 & 45 & 27 & 14 & 21 & 99 & 43 & 3.53 & 86.0 & 0.4 & 5.1 & 6.7 & 34.7 & 26.1 & 39.5 & 2.37 & 1.3 & 44.0 & 8.31 & 52.8 & 3.92 & 930 \\
\hline $4 \cdot 2,57 \cdot 60$ & 354 & 12 & 32 & 6 & 73 & 56 & 12 & 12 & 12 & ${ }^{83}$ & 41 & 2.93 & 35.7 & 0.85 & 4.6 & 5.8 & 25.0 & 11.5 & 27,9 & 1.6 & 0.01 & 49.0 & 7.0 & 28.9 & 2.07 & 378 \\
\hline $\begin{array}{l}5-1,81-85 \\
6=1,30.33\end{array}$ & 391 & 24 & 38 & 23 & 77 & 70 & 16 & 18 & 26 & 103 & 56 & 2.27 & 53.0 & 0.85 & 5.3 & 4.7 & 20.7 & 19.3 & 32.9 & 1.8 & 0.9 & 46.0 & 7.8 & 34.8 & 2.96 & 467 \\
\hline $6-1,30-33$ & 438 & 24 & 24 & 22 & 42 & 82 & 17 & 14 & 40 & 122 & 88 & 3.14 & 103 & 0.95 & 6.1 & 5.4 & 26.7 & 21.8 & 40.7 & 1.6 & 0.47 & 89.0 & 17.9 & 41.2 & 9.0 & 940 \\
\hline $9-2,143-146$ & 469 & 10 & $\begin{array}{l}40 \\
52\end{array}$ & $\begin{array}{l}4 \\
5\end{array}$ & $\begin{array}{l}42 \\
40\end{array}$ & $\begin{array}{l}35 \\
49\end{array}$ & 12 & 9 & 20 & $\begin{array}{l}75 \\
60\end{array}$ & 32 & $\begin{array}{l}2.88 \\
257\end{array}$ & $\begin{array}{l}24.0 \\
260\end{array}$ & 0.4 & 4.3 & 4.3 & 19.8 & 10.0 & 24.2 & 1.6 & 0.25 & 33.4 & 55 & $\begin{array}{r}24.3 \\
18.9\end{array}$ & 2.44 & $\begin{array}{l}224 \\
370\end{array}$ \\
\hline $\begin{array}{l}10-1.101-105 \\
10-43-46\end{array}$ & $\begin{array}{l}486 \\
487\end{array}$ & $\begin{array}{l}10 \\
10\end{array}$ & $\begin{array}{l}52 \\
46\end{array}$ & $\begin{array}{l}5 \\
4\end{array}$ & $\begin{array}{l}40 \\
42\end{array}$ & $\begin{array}{l}49 \\
55\end{array}$ & 12 & 11 & $\begin{array}{r}18 \\
5\end{array}$ & $\begin{array}{l}60 \\
49\end{array}$ & $\begin{array}{l}31 \\
20\end{array}$ & $\begin{array}{l}2.57 \\
2.42\end{array}$ & $\begin{array}{l}26.0 \\
22.7\end{array}$ & 0.41 & $\begin{array}{l}2.92 \\
3.3\end{array}$ & $\begin{array}{l}2.8 \\
3.2\end{array}$ & $\begin{array}{l}14.5 \\
18.9\end{array}$ & 10.1 & 18.3 & 1.3 & 0.01 & & 5.2 & $\begin{array}{r}18.9 \\
186\end{array}$ & $\begin{array}{l}1.70 \\
0.89\end{array}$ & 370 \\
\hline $\begin{array}{l}10-2,43-46 \\
11-2,45-49\end{array}$ & $\begin{array}{l}487 \\
506\end{array}$ & $\begin{array}{l}10 \\
10\end{array}$ & $\begin{array}{l}46 \\
40\end{array}$ & $\begin{array}{l}4 \\
4\end{array}$ & $\begin{array}{l}42 \\
50\end{array}$ & $\begin{array}{l}55 \\
85\end{array}$ & $\begin{array}{l}12 \\
12\end{array}$ & $\begin{array}{l}9 \\
7\end{array}$ & $\begin{array}{r}5 \\
18\end{array}$ & $\begin{array}{l}49 \\
55\end{array}$ & $\begin{array}{l}20 \\
23\end{array}$ & $\begin{array}{r}2,42 \\
2,19\end{array}$ & $\begin{array}{l}22.7 \\
2664\end{array}$ & 0.17 & $\begin{array}{l}3.3 \\
3.9\end{array}$ & 3.2 & $\begin{array}{l}18.9 \\
18.6\end{array}$ & $\begin{array}{l}10.4 \\
13.3\end{array}$ & $\begin{array}{l}18.1 \\
22.7\end{array}$ & $\begin{array}{l}1.5 \\
1.37\end{array}$ & $\begin{array}{l}0.9 \\
1.5\end{array}$ & $\begin{array}{l}2558 \\
257\end{array}$ & $\begin{array}{l}4.30 \\
4.20\end{array}$ & $\begin{array}{l}18.6 \\
25.4\end{array}$ & 0.89 & 272 \\
\hline $11-3,24-32$ & 507 & 12 & 49 & 7 & 56 & 64 & 11 & 10 & 12 & 55 & 22 & 2.97 & 11.0 & 0.1 & 3.46 & 4.1 & $\begin{array}{l}18.6 \\
22.4\end{array}$ & $\begin{array}{l}13.3 \\
12.9\end{array}$ & $\begin{array}{l}22.7 \\
20.8\end{array}$ & 1.37 & 0.44 & $\begin{array}{l}25.7 \\
31.9\end{array}$ & $\begin{array}{l}4.20 \\
5.35\end{array}$ & $\begin{array}{l}25.4 \\
24.8\end{array}$ & $\begin{array}{l}1.66 \\
0.64\end{array}$ & $\begin{array}{l}331 \\
348\end{array}$ \\
\hline $11-4,17-24$ & 508 & 11 & 10 & 7 & 58 & 40 & 12 & 14 & 16 & 55 & 28 & 3.12 & 35.7 & 1.5 & 4.5 & 2.8 & 18.6 & 23.8 & 24.3 & 1.35 & 0.9 & 34.7 & $\begin{array}{l}5.35 \\
6.37\end{array}$ & $\begin{array}{l}25.8 \\
25.2\end{array}$ & 0.64 & $\begin{array}{l}348 \\
510\end{array}$ \\
\hline $11-6,109.115$ & 512 & 18 & 14 & 105 & 120 & 94 & 47 & 40 & 162 & 190 & 245 & 3.39 & 132 & 0.4 & 13.9 & 8.5 & 24.7 & 82.7 & 84.3 & 4.4 & 2.7 & 125.0 & 17.5 & 30.3 & 8.5 & 670 \\
\hline $13-2$ & 537 & 10 & 110 & 33 & 140 & 45 & 17 & 23 & 71 & 110 & 65 & & & & & & & & & & & & & & & \\
\hline Site 323 & & & & & & & & & & & & & & & & & & & & & & & & & & \\
\hline $1-1,50-60$ & 76 & 8 & 40 & 3 & 29 & 54 & & 2 & 15 & 47 & 16 & 8.7 & 98.8 & 0.30 & 3.5 & 1.3 & 7.8 & 49.3 & 18.2 & 1.01 & 0.71 & 32.8 & 4.5 & 13.6 & 3.4 & 551 \\
\hline $1-1,140-150$ & 77 & 27 & 33 & 13 & 120 & 68 & & $?$ & 21 & 114 & 106 & 4.2 & 119.0 & 0.9 & 6.4 & 3.2 & 15.0 & 13.3 & 29.7 & 1.3 & 1.63 & 54.1 & 10.6 & 19.1 & 22.4 & 438 \\
\hline $1-2,34-43$ & 79 & 29 & 25 & 19 & 150 & 80 & & 12 & 34 & 115 & 46 & 4.2 & 155.0 & 0.50 & 5.7 & 5.1 & 21.7 & 15. & 27.7 & 1.1 & 1.52 & 69.2 & 12.9 & 31.8 & 32.3 & 906 \\
\hline $1-3,78-88$ & 80 & 23 & 27 & 18 & 200 & 75 & & 12 & 30 & 115 & 34 & 3.4 & 23.9 & 0.20 & 4.5 & 3.8 & 17.0 & 18.9 & 20.2 & 1.16 & 0.85 & 48.0 & 7.1 & 18.5 & 10.5 & 754 \\
\hline $1-4,118-127$ & 81 & 24 & 24 & 16 & 200 & 120 & & 8 & 33 & 108 & 60 & 3.2 & 160.0 & 0.90 & 6.1 & 4.1 & 25.0 & 18.2 & 29.4 & 1.10 & 2.00 & 65.0 & 10.5 & 28.1 & 22.0 & 119 \\
\hline $3-1,88-94$ & 257 & 15 & 44 & 10 & 200 & 110 & & 5 & 15 & 108 & 22 & 2.2 & 122.5 & 0.3 & 4.8 & 3.0 & 13.3 & 8.7 & 21.2 & 1.2 & 0.60 & 42.2 & 9.5 & 9.3 & 20.4 & 464 \\
\hline $3-2,14-20$ & 258 & 12 & 70 & 4 & 64 & 150 & & 2 & 17 & 55 & 14 & 4.3 & 91.3 & 0.3 & 3.8 & 1.3 & 6.1 & 3.9 & 16.1 & 0.6 & 0.40 & 31.5 & 4.4 & 8.1 & 3.9 & 364 \\
\hline $3-2,61 \cdot 70$ & 258 & 24 & 21 & 21 & 170 & 120 & & 10 & 26 & 142 & 83 & 2.9 & 154 & 0.4 & 5.4 & 4.2 & 20.5 & 11.8 & 12.4 & 1.1 & & 49.1 & 8.3 & 35.3 & 20.5 & 926 \\
\hline & 315 & 14 & 78 & 3 & 24 & 26 & & 2 & 6 & 62 & 16 & 4.6 & & 0.30 & 1.8 & 2.1 & 8.5 & 6.6 & 16.6 & 0.8 & & 36.7 & 6.0 & 9.6 & 3.7 & 659 \\
\hline $7 \cdot 2,105-110$ & 363 & 16 & 30 & 28 & 140 & 90 & & 14 & 50 & 92 & 113 & 4.1 & 112.2 & 0.5 & 6.0 & 4.5 & 17.0 & 15.5 & 29.9 & 1.3 & & 60.5 & 9.1 & 33.2 & 14.9 & 976 \\
\hline $7-3,18-24$ & 364 & 19 & 21 & 19 & 140 & $\begin{array}{r}80 \\
40\end{array}$ & & 12 & 34 & 115 & 94 & 3.7 & 159 & 0.28 & 6.4 & 4.5 & 20.1 & 13.5 & 34.7 & 1.61 & 0.59 & 76.2 & 9.7 & 48.8 & 22.9 & 789 \\
\hline $\begin{array}{l}10-1,113-122 \\
10-2,75-85\end{array}$ & $\begin{array}{l}504 \\
505\end{array}$ & 18 & 31 & $\begin{array}{l}14 \\
19\end{array}$ & $\begin{array}{l}220 \\
180\end{array}$ & $\begin{array}{r}140 \\
60\end{array}$ & & 45 & $\begin{array}{l}26 \\
38\end{array}$ & $\begin{array}{l}105 \\
97\end{array}$ & 90 & $\begin{array}{l}34.6 \\
3.5\end{array}$ & $\begin{array}{l}111.0 \\
1370\end{array}$ & 0.44 & 5.0 & 3.8 & 22.1 & 11.1 & 23.1 & & 0.71 & 63.2 & 8.7 & 35 & 12.1 & 603 \\
\hline $\begin{array}{l}10-2,75-85 \\
10-3,106-114\end{array}$ & $\begin{array}{l}505 \\
507\end{array}$ & $\begin{array}{l}19 \\
16\end{array}$ & $\begin{array}{l}22 \\
27\end{array}$ & $\begin{array}{l}19 \\
12\end{array}$ & $\begin{array}{l}180 \\
150\end{array}$ & $\begin{array}{r}60 \\
110\end{array}$ & & $\begin{array}{l}15 \\
10\end{array}$ & $\begin{array}{l}38 \\
16\end{array}$ & $\begin{array}{l}97 \\
94\end{array}$ & $\begin{array}{l}305 \\
48\end{array}$ & $\begin{array}{l}3.5 \\
4.1\end{array}$ & $\begin{array}{l}137.0 \\
157.5\end{array}$ & $\begin{array}{l}0.3 \\
0.53\end{array}$ & $\begin{array}{l}4.4 \\
4.5\end{array}$ & $\begin{array}{l}4.3 \\
4.9\end{array}$ & $\begin{array}{l}5.9 \\
20.9\end{array}$ & 19.5 & $\begin{array}{l}18.1 \\
22.6\end{array}$ & $\begin{array}{l}0.90 \\
1.43\end{array}$ & $\begin{array}{l}1.39 \\
1.05\end{array}$ & $\begin{array}{l}34.5 \\
46.3\end{array}$ & $\begin{array}{l}7.9 \\
7.6\end{array}$ & 159 & 16.6 & $\begin{array}{l}373 \\
513\end{array}$ \\
\hline $11-1,22-32$ & 551 & 22 & 36 & 8 & 210 & 90 & & 4 & 26 & 105 & 39 & 1.5 & 180.5 & 0.40 & $\begin{array}{l}4.9 \\
6.6\end{array}$ & 3.7 & 14.7 & $\begin{array}{r}11.4 \\
9.7\end{array}$ & $\begin{array}{l}2.6 \\
32.9\end{array}$ & $\begin{array}{l}1.43 \\
1.29\end{array}$ & $\begin{array}{l}1.05 \\
2.06\end{array}$ & $\begin{array}{r}46.3 \\
6.76\end{array}$ & $\begin{array}{r}7.6 \\
10.3\end{array}$ & $\begin{array}{l}25 \\
63\end{array}$ & $\begin{array}{l}29.0 \\
29.1\end{array}$ & $\begin{array}{l}513 \\
916\end{array}$ \\
\hline $11-2,137-148$ & 553 & 25 & 35 & 8 & 150 & 110 & & 6 & 25 & 97 & 36 & 4.8 & 131.3 & 0.7 & 6.0 & 2.6 & 13.4 & 11.4 & 25.8 & 1.0 & 0.9 & 56.9 & 10.0 & $\begin{array}{l}63 \\
30.9\end{array}$ & $\begin{array}{l}29.1 \\
19.0\end{array}$ & $\begin{array}{l}916 \\
437\end{array}$ \\
\hline $13-5,106-115$ & 624 & 27 & 27 & 27 & 120 & 110 & & 15 & 45 & 135 & 51 & 6.5 & 130.1 & 0.67 & 6.7 & 4.8 & 20.6 & 18.8 & 35.1 & 1.66 & 0.86 & 63.9 & 8.5 & 25.5 & 15.9 & 425 \\
\hline $13-6,145-150$ & 625 & ii & 31 & 20 & 120 & 180 & & 12 & 62 & 85 & 44 & 5.9 & 124.2 & 0.66 & 6.4 & 3.7 & 15.1 & 12.9 & 30.7 & 1.20 & 0.26 & 58.6 & 8.5 & 22.9 & 14.2 & 436 \\
\hline $14.2,1-8$ & 637 & 16 & 40 & 36 & 180 & 180 & & 18 & 42 & 85 & 60 & 5.3 & 133.5 & 0.30 & 6.3 & 3.4 & 13.7 & 16.3 & 26.9 & 1.6 & 1.27 & 58.7 & 9.6 & 4.8 & 13.7 & 269 \\
\hline $14-2,128-135$ & 638 & 17 & 18 & 180 & 290 & 150 & & 58 & 220 & 205 & 372 & 6.4 & 54.3 & 0.40 & 20.1 & 7.0 & 36.6 & 64 & 83.1 & 5.6 & 2.49 & 143 & 10.3 & 14.9 & 41.5 & 655 \\
\hline $15-1,52-60$ & 656 & 17 & 20 & 160 & 180 & 80 & & 23 & 325 & 310 & 340 & 6.8 & 49.7 & 0.30 & 19.8 & 8.9 & 35.5 & 75.9 & 89.0 & 5.2 & 3.53 & 149 & 9.3 & 5.7 & 27.4 & 789 \\
\hline $15-2,91-100$ & 657 & 15 & 20 & 96 & 240 & 78 & & 23 & 195 & 230 & 195 & 5.1 & 63.4 & 0.30 & 11.6 & 6.3 & 24.6 & 42.8 & 59.3 & 2.7 & 2.40 & 94.8 & 9.0 & 6.9 & 17.8 & 354 \\
\hline $15-3,29-36$ & 658 & 10 & 20 & 74 & 310 & 100 & & 22 & 127 & 210 & 194 & 6.2 & 124.3 & 0.43 & 10.9 & 6.4 & 26.3 & 35.6 & 58.2 & 2.67 & 2.35 & 106.7 & 7. & 11.1 & 4.4 & 502 \\
\hline $15-4,54$ & 660 & 8 & 20 & 82 & 340 & 65 & & 25 & 140 & 230 & 190 & 3.6 & 89.9 & 0. & 10.0 & 5.5 & 22. & 34 & 46.1 & 1.8 & 1.8 & 82 & 7.7 & & 4.8 & 259 \\
\hline $15-5,88$ & 661 & 13 & 2 & 49 & 190 & 56 & & 20 & 109 & 230 & 197 & 3.5 & 84 & 0. & 9.2 & 5.5 & 19. & 33.8 & 43 & & 1. & 84 & 10.0 & 27.5 & 7.3 & 708 \\
\hline $15-6.1$ & 663 & 4 & 20 & 36 & 280 & 60 & & 12 & 63 & 170 & 120 & 2.3 & 74 & 0.2 & 6.5 & 1.4 & 11.0 & 20.5 & 31.4 & & 0. & 49 & 6. & & 3.7 & 262 \\
\hline & 665 & 5 & 15 & 28 & 210 & 65 & & 2 & 42 & 149 & 104 & 2.9 & 72. & 0.36 & 5.9 & 4.4 & & & & 1.52 & 1.2 & 47 & & & 8 & 744 \\
\hline & 66 & 15 & 2 & 34 & 130 & 46 & & 17 & 84 & 190 & 148 & 5.4 & 76.3 & 0. & 6.5 & 6.6 & 24 & 38.5 & 34 & 2. & 1. & 89 & 5 & 21 & 2.9 & 148 \\
\hline & 669 & 16 & 2 & 29 & 120 & 54 & & 12 & 63 & 210 & 163 & 4.9 & 70.2 & 0. & 7.8 & 4.0 & 23 & 21 & 42. & & 0. & 60 & 5. & & 5.11 & 182 \\
\hline & 695 & 20 & 1 & 28 & 67 & 38 & & 8 & 42 & 180 & 117 & 4.6 & & 0. & 6.8 & 4.4 & & & 42. & & 0. & & 9.4 & 31 & 10.8 & 1302 \\
\hline $18-3,130-$ & 697 & 25 & 16 & 17 & $\begin{array}{r}70 \\
10\end{array}$ & $\begin{array}{l}71 \\
78\end{array}$ & & 6 & $\begin{array}{l}38 \\
50\end{array}$ & 180 & 163 & 4.5 & 77.3 & 0. & 6.6 & 6.0 & 23 & 15 & 37 & 1.8 & 0. & 66. & & & 18.0 & $\begin{array}{r}4176 \\
0560\end{array}$ \\
\hline $18-4.115-124$ & 699 & 26 & 19 & 34 & 110 & $\begin{array}{l}78 \\
75\end{array}$ & & 4 & 50 & $\begin{array}{l}200 \\
195\end{array}$ & $\begin{array}{l}485 \\
495\end{array}$ & 6.0 & 60.6 & 0.48 & 8.1 & 5.6 & 31.6 & 16.4 & 44.3 & 2.49 & 2.31 & 47.0 & & 36.2 & 3.9 & 10560 \\
\hline $18-5,65-73$ & 700 & 14 & 20 & 49 & 180 & 75 & & 5 & 102 & 195 & 495 & 0.3 & 61.6 & 0.3 & 10.5 & 5.7 & 21.9 & 20.5 & 28.5 & 2.9 & 0.30 & 62.4 & 5.6 & 33.9 & 1.7 & 1204 \\
\hline Site 324 & & & & & & & & & & & & & & & & & & & & & & & & & & \\
\hline $1 \cdot 2,126$ & 11 & 14 & 35 & 13 & 56 & 78 & 18 & 10 & 25 & 102 & 56 & 5.33 & 138 & 1.65 & 7.9 & 3.6 & 16.2 & 15.2 & 48.5 & 1.69 & 0.081 & 77.2 & 15.0 & 34.1 & & 1040 \\
\hline & 13 & 14 & 32 & 12 & 54 & 46 & 17 & 3 & 28 & 103 & 30 & 5.4 & 150 & & 6.65 & 4.1 & & 11. & & 1.54 & & 80 & 18. & & 6.5 & 750 \\
\hline & 15 & 13 & 35 & 9 & 58 & 65 & 16 & 5 & 30 & 115 & 14 & 4.98 & 142 & 0.7 & 8.65 & 4.8 & 17.7 & 14 & 51. & 1.86 & 0. & 85 & 21 & 34. & 7.0 & 730 \\
\hline & 17 & 9 & 45 & 9 & 52 & 78 & 21 & 9 & 13 & 85 & 23 & 6.72 & 13 & 0.9 & 7.9 & 3.8 & 16 & 12 & 44 & 2.0 & & 74 & 18 & 33 & 6.5 & 740 \\
\hline & 48 & 15 & 37 & 12 & 46 & 100 & 24 & 9 & 13 & 125 & 13 & 8.8 & 64 & 2.2 & 12.7 & 4.1 & 14 & 14 & 76 & 2. & 0. & 121. & 32 & 45.1 & 14.8 & 78 \\
\hline & 49 & 16 & 35 & 12 & 46 & 80 & 23 & 6 & 17 & 118 & 8 & 7.4 & 16 & 2.2 & 14.4 & 3.8 & 13 & 14 & 75 & 2. & 0. & 124. & 33. & 53.4 & 14. & 670 \\
\hline & 50 & 14 & 45 & 16 & 68 & 68 & 23 & 8 & 28 & 112 & 28 & 6.1 & 15 & & 9. & 4.6 & 17 & & 51 & 1. & & 95 & 23. & & 10.6 & 8. \\
\hline & 52 & 12 & 35 & 10 & 40 & 73 & 21 & 5 & 15 & 103 & 17 & 7.1 & 14 & 2.2 & 9.45 & 4. & & 13 & 58 & & & 101 & 23 & & 10.4 & 6 \\
\hline & 53 & 12 & 33 & 10 & 48 & 53 & 21 & 6 & 14 & 118 & 23 & 6. & is & 1.7 & 11.5 & 3 & is & & 67 & 2. & 0 . & 107 & 28 & & 9.1 & 5 \\
\hline & 55 & 10 & 39 & 8 & 44 & 58 & 19 & 6 & 28 & 11 & 18 & 6. & 12 & 1.44 & 9. & 3.3 & & & & & & 96 & 23 & & 8.9 & \\
\hline & 77 & 12 & 32 & 9 & 44 & 60 & 23 & 7 & 12 & 10 & 10 & & & 2 & & 3 & & & & & 0.02 & & & & 10.5 & 90 \\
\hline & 79 & 12 & 4. & 10 & 54 & 68 & 23 & 8 & 36 & 104 & 19 & & 1 & 2. & 11 & 3 & 15. & 12. & 65 & $\begin{array}{l}1.92 \\
1.97\end{array}$ & 0.03 & $\begin{array}{r}99.0 \\
1040\end{array}$ & 26.1 & $\begin{array}{l}46.7 \\
43.7\end{array}$ & $\begin{array}{l}8.6 \\
8.6\end{array}$ & $\begin{array}{l}670 \\
7900\end{array}$ \\
\hline & 80 & 18 & 44 & 10 & 52 & 94 & 19 & 14 & 14 & 11 & 14 & & 13 & 1. & 11.9 & & $\begin{array}{l}18.9 \\
19.6\end{array}$ & $\begin{array}{l}16.5 \\
14.2\end{array}$ & $\begin{array}{l}69.0 \\
62.2\end{array}$ & $\begin{array}{l}1.97 \\
2.27\end{array}$ & & $\begin{array}{l}104.0 \\
106.0\end{array}$ & $\begin{array}{l}25.1 \\
25.2\end{array}$ & $\begin{array}{l}43.7 \\
51.0\end{array}$ & $\begin{array}{r}8.6 \\
10.6\end{array}$ & $\begin{array}{l}790 \\
650\end{array}$ \\
\hline & 81 & 12 & 39 & 12 & 37 & 45 & 20 & 4 & 15 & 110 & 15 & 6.60 & 132 & & 10.2 & 4.8 & 19.6 & $\begin{array}{l}14.2 \\
166\end{array}$ & $\begin{array}{l}62.2 \\
82.0\end{array}$ & 2.2 & & $\begin{array}{l}106.0 \\
102.0\end{array}$ & $\begin{array}{l}28.2 \\
28.3\end{array}$ & $\begin{array}{l}1.0 \\
46.0\end{array}$ & $\begin{array}{l}11.0 \\
11.1\end{array}$ & $\begin{array}{r}650 \\
1610\end{array}$ \\
\hline $\begin{array}{l}3-5,99-110 \\
3-6,138-148\end{array}$ & $\begin{array}{l}83 \\
85\end{array}$ & $\begin{array}{l}16 \\
10\end{array}$ & $\begin{array}{l}45 \\
32\end{array}$ & $\begin{array}{l}12 \\
12\end{array}$ & $\begin{array}{l}37 \\
48\end{array}$ & $\begin{array}{l}60 \\
62\end{array}$ & $\stackrel{21}{18}$ & $\begin{array}{l}6 \\
4\end{array}$ & $\begin{array}{r}9 \\
12\end{array}$ & $\begin{array}{l}116 \\
110\end{array}$ & $\begin{array}{r}9 \\
30\end{array}$ & $\begin{array}{l}6.8 \\
5.0\end{array}$ & $\begin{array}{l}138 \\
78.0\end{array}$ & $\begin{array}{l}0.95 \\
1.08\end{array}$ & $\begin{array}{c}13.7 \\
8.05\end{array}$ & $\begin{array}{l}4.4 \\
4.6\end{array}$ & $\begin{array}{l}20.1 \\
19.0\end{array}$ & $\begin{array}{l}16.6 \\
12.1\end{array}$ & $\begin{array}{l}82.0 \\
47.1\end{array}$ & $\begin{array}{l}1.9 \\
1.98\end{array}$ & $\begin{array}{l}2.1 \\
0.56\end{array}$ & $\begin{array}{r}102.0 \\
81.0\end{array}$ & $\begin{array}{l}28.3 \\
16.1\end{array}$ & $\begin{array}{l}46.0 \\
39.8\end{array}$ & $\begin{array}{r}11.1 \\
5.4\end{array}$ & 860 \\
\hline $\begin{array}{l}3-6,138-148 \\
4-2,113-123\end{array}$ & $\begin{array}{r}85 \\
107\end{array}$ & $\begin{array}{l}10 \\
13\end{array}$ & 43 & 13 & $\begin{array}{l}48 \\
54\end{array}$ & $\begin{array}{l}62 \\
66\end{array}$ & 24 & $\begin{array}{l}4 \\
9\end{array}$ & $\begin{array}{l}12 \\
16\end{array}$ & $\begin{array}{l}100 \\
368\end{array}$ & $\begin{array}{l}30 \\
10\end{array}$ & 5.21 & $\begin{array}{l}134.0 \\
134\end{array}$ & 2.2 & $\begin{array}{l}8.00 \\
9.0\end{array}$ & $\begin{array}{l}4.6 \\
3.4\end{array}$ & $\begin{array}{l}19.0 \\
19.5\end{array}$ & $\begin{array}{l}13.1 \\
13.6\end{array}$ & $\begin{array}{l}4.1 \\
67.0\end{array}$ & $\begin{array}{l}1.98 \\
2.4\end{array}$ & $\begin{array}{l}0.56 \\
0.1\end{array}$ & $\begin{array}{l}81.0 \\
102\end{array}$ & 22.1 & 45.0 & 9.7 & 494 \\
\hline
\end{tabular}




\begin{tabular}{|c|c|c|c|c|c|c|c|c|c|c|c|c|c|c|c|c|c|c|c|c|c|c|c|c|c|c|}
\hline $4-3,30-40$ & 108 & 9 & 45 & 13 & 46 & 53 & 17 & 7 & 18 & 119 & 23 & 5.28 & 124.0 & 2.9 & 10.5 & 4.34 & 19.4 & 17.6 & 60.0 & 2.1 & 0.49 & 110.0 & 24.8 & 38.0 & 9.6 & 600 \\
\hline $4-4,54-64$ & 109 & 12 & 43 & 13 & 46 & 53 & 24 & 6 & 20 & 113 & 17 & 6.76 & 124.0 & 3.1 & 13.7 & 4.5 & 21.8 & 18.4 & 83.1 & 2.2 & 1.2 & 118 & 22.5 & 47.2 & 8.9 & 1300 \\
\hline $5-2,66-83$ & 135 & 25 & 35 & 7 & 47 & 85 & 18 & 11 & 5 & 112 & 24 & 5.32 & 111.0 & 2.2 & 9.3 & 4.65 & 19.8 & 13.2 & 55.4 & 2.0 & 1.44 & 90.0 & 22.5 & & 8.0 & 550 \\
\hline $5-3,25-35$ & 136 & 22 & 35 & 5 & 34 & 110 & 19 & 9 & 4 & 107 & 11 & 7.40 & 161 & 2.4 & 10.0 & 3.6 & 13.1 & 11.9 & 76.1 & 1.95 & 0.01 & 118 & 28.8 & 44.1 & 10.3 & 750 \\
\hline $6-2,19-30$ & 144 & 27 & 35 & 11 & 39 & 110 & 19 & 12 & 10 & 108 & 23 & 3.10 & 164 & 2.0 & 11.9 & 4.9 & 18.0 & 16.6 & 77.4 & 2.7 & 1.76 & 105 & 30.3 & 49.8 & 12.0 & 560 \\
\hline $7 \cdot 1.69 .78$ & 153 & 15 & 14 & 7 & 42 & 87 & 15 & 12 & 20 & 119 & 18 & 5.60 & 156 & 1.05 & 11.5 & 4.8 & 18.5 & 19.1 & 73.1 & 2.8 & 1.5 & 100 & 29.2 & 54.0 & 12.0 & 640 \\
\hline $\begin{array}{l}7-3,110-121 \\
7-6.119 .129\end{array}$ & $\begin{array}{l}156 \\
161\end{array}$ & 20 & 43 & 16 & $\begin{array}{l}52 \\
47\end{array}$ & $\begin{array}{l}94 \\
90\end{array}$ & $\begin{array}{l}19 \\
16\end{array}$ & 16 & 16 & ${ }_{120}^{130}$ & 20 & $\begin{array}{l}4.73 \\
450\end{array}$ & $\begin{array}{l}160 \\
150\end{array}$ & 1.7 & $\begin{array}{l}10.4 \\
10.4\end{array}$ & 5.1 & 18.1 & 19.8 & $\begin{array}{l}67.1 \\
65.3\end{array}$ & 2.3 & 1.3 & 95 & $\begin{aligned} 26.4 \\
28.5\end{aligned}$ & & & 520 \\
\hline $\begin{array}{l}7-6.1199 .129 \\
8 \cdot 3,61-71\end{array}$ & $\begin{array}{l}161 \\
174\end{array}$ & $\begin{array}{l}13 \\
17\end{array}$ & $\begin{array}{l}37 \\
36\end{array}$ & $\begin{array}{l}15 \\
18\end{array}$ & $\begin{array}{l}47 \\
56\end{array}$ & $\begin{array}{r}90 \\
110\end{array}$ & $\begin{array}{l}16 \\
19\end{array}$ & $\begin{array}{l}13 \\
14\end{array}$ & $\begin{array}{l}20 \\
17\end{array}$ & $\begin{array}{l}1122 \\
119\end{array}$ & $\begin{array}{l}20 \\
15\end{array}$ & $\begin{array}{l}4.50 \\
3.67\end{array}$ & $\begin{array}{l}150 \\
141\end{array}$ & $\begin{array}{l}1.8 \\
1.4\end{array}$ & $\begin{array}{r}10.1 \\
9.4\end{array}$ & $\begin{array}{l}4.2 \\
4.3\end{array}$ & $\begin{array}{l}15.8 \\
15.8\end{array}$ & $\begin{array}{l}19.7 \\
15.4\end{array}$ & $\begin{array}{r}65.3 \\
57.0\end{array}$ & $\begin{array}{l}1.97 \\
1.86\end{array}$ & $\begin{array}{l}0.6 \\
0.74\end{array}$ & $\begin{array}{l}104 \\
93.0\end{array}$ & $\begin{array}{l}28.5 \\
26.9\end{array}$ & $\begin{array}{l}48.7 \\
48.5\end{array}$ & $\begin{array}{l}10.4 \\
9.3\end{array}$ & $\begin{array}{l}540 \\
580\end{array}$ \\
\hline \multicolumn{27}{|l|}{ Site 325} \\
\hline $1-1,49-59$ & 34 & 9 & 28 & 12 & 130 & 46 & & 10 & & 97 & 18 & 3.2 & 73.4 & 0.4 & 4.2 & 4.7 & 21.6 & 11.8 & 15.1 & 1.0 & 0.60 & 33.6 & 6.0 & 31.3 & 9.6 & 378 \\
\hline $1-1,133-144$ & 35 & 10 & 50 & 15 & 75 & 46 & 17 & 11 & & 118 & 41 & 4.6 & 100 & 0.5 & 4.6 & 5.0 & 24.2 & 17.4 & 19.1 & 1.4 & 0.45 & 34.5 & 4.8 & 22.9 & 7.9 & 534 \\
\hline $1-2,26-36$ & 36 & 9 & 56 & 14 & 56 & 36 & 10 & 5 & & 118 & 26 & 5.5 & 111.0 & 0.03 & & 4.8 & 18.7 & 16.8 & 20.0 & 0.8 & 0.5 & 42.6 & 5.0 & 21.6 & 11.0 & 622 \\
\hline $1-4,81-92$ & 39 & 12 & 39 & 12 & 64 & 41 & 18 & 7 & & 105 & 26 & 3.5 & 90.1 & 0.2 & 4.0 & 3.8 & 19.0 & 11.6 & 17.7 & 1.1 & 0.99 & 34.1 & 5.4 & 29.4 & 6.6 & 450 \\
\hline 2-2, $39-49$ & 168 & 9 & 46 & 9 & 52 & 46 & 16 & 5 & & 94 & 26 & 4.6 & 62.3 & 0.2 & 3.9 & 3.6 & 16.7 & 7.1 & 13.5 & 1.1 & 0.40 & 32.9 & 3.5 & & 1.7 & 366 \\
\hline $3 \cdot 2,11-24$ & 178 & 8 & 52 & 13 & 60 & 46 & 17 & 8 & & 97 & 25 & 2.6 & 56.3 & 0.3 & 3.8 & 3.8 & 18.9 & 10.3 & 11.7 & 0.9 & 0.55 & 32.0 & 3.9 & 35.9 & 1.5 & 141 \\
\hline $3-3,76-87$ & 180 & 8 & 48 & 7 & 56 & 66 & 16 & 6 & & 92 & 23 & 3.0 & 52.2 & 0.4 & 3.8 & 3.2 & 17.1 & 6.9 & 13.9 & 0.9 & & 23.7 & 3.8 & 22.3 & 5.4 & 307 \\
\hline $3-4,26-36$ & 182 & 9 & 39 & 9 & 60 & 47 & 17 & 5 & & 94 & 24 & 3.3 & 74.4 & 0.2 & 3.4 & 3.3 & 17.6 & 7.5 & 11.9 & 0.7 & & & 3.8 & 27.0 & 5.8 & 339 \\
\hline$-2,90$ & 293 & 10 & 53 & 8 & 40 & 32 & 14 & 6 & & 92 & 26 & 3.9 & 50.9 & 0.3 & 3.0 & 3.8 & 19.4 & 10.5 & 11.9 & 1.0 & 0. & 33.3 & 4.1 & 27.1 & 1.8 & 382 \\
\hline $4+3,20-26$ & 294 & 12 & 52 & 8 & 56 & 36 & 17 & 8 & & 97 & 30 & 8.2 & 1.4 & 0.28 & 3.5 & 4.7 & 13.7 & 12.9 & 14.0 & 1.29 & 4. & 48.2 & 4.1 & 21.0 & 14.2 & 249 \\
\hline $\begin{array}{l}4,130 \\
-3,130\end{array}$ & 295 & 7 & 53 & 8 & 45 & 32 & 14 & 5 & & 90 & 27 & 2.9 & 45.8 & 0.09 & 3.5 & 3.1 & 17.8 & 11.3 & 12.0 & 0.8 & 0. & 29.4 & 3.9 & 23.9 & 1.1 & 273 \\
\hline $4, C C$ & 295 & 10 & 20 & $?$ & 36 & $\begin{array}{c}22 \\
50\end{array}$ & 10 & 5 & & 103 & 33 & 4.1 & 49.5 & 0.2 & 3.5 & 4.0 & 16.1 & 11.5 & 10.4 & 0.9 & 0.64 & 35.2 & 4.1 & 82.4 & 23.6 & 341 \\
\hline $5-1,55-57$ & 404 & ${ }_{13}^{13}$ & $\begin{array}{l}35 \\
26\end{array}$ & 10 & & 50 & 14 & $\begin{array}{l}5 \\
8\end{array}$ & & 105 & 48 & $\begin{array}{l}4.6 \\
2.9\end{array}$ & $\begin{array}{l}63.7 \\
80.5\end{array}$ & 0.3 & 4.8 & 4.7 & 21.2 & 8.8 & 19.9 & 1.0 & 7.1 & $\begin{array}{l}48.5 \\
46.5\end{array}$ & 6.8 & 1.9 & 9.1 & 566 \\
\hline $8-1,45-49$ & $\begin{array}{l}613 \\
615\end{array}$ & ${ }_{12}^{12}$ & $\begin{array}{l}26 \\
30\end{array}$ & $\begin{array}{r}10 \\
8\end{array}$ & $\begin{array}{l}70 \\
82\end{array}$ & $\begin{array}{l}39 \\
44\end{array}$ & $\begin{array}{l}16 \\
18\end{array}$ & $\begin{array}{l}8 \\
6\end{array}$ & & $\begin{array}{l}87 \\
69\end{array}$ & 39 & $\begin{array}{l}2.9 \\
3.4\end{array}$ & $\begin{array}{r}80.5 \\
1060\end{array}$ & 0.4 & 47 & 4.1 & 19.3 & 10.9 & 17.9 & 1.0 & 0.78 & 46.5 & 3.1 & 20.5 & 8.8 & 281 \\
\hline $3-3,27-35$ & $\begin{array}{l}613 \\
616\end{array}$ & 12 & 35 & $10^{\circ}$ & $\begin{array}{l}82 \\
70\end{array}$ & $\begin{array}{l}44 \\
40\end{array}$ & $\begin{array}{l}18 \\
15\end{array}$ & $\begin{array}{l}6 \\
7\end{array}$ & & $\begin{array}{r}69 \\
115\end{array}$ & $\begin{array}{l}46 \\
43\end{array}$ & $\begin{array}{l}3.4 \\
2.8\end{array}$ & $\begin{array}{r}106.0 \\
90.2\end{array}$ & 0.20 & $\begin{array}{l}4.7 \\
4.3\end{array}$ & $\begin{array}{l}3.6 \\
4.3\end{array}$ & $\begin{array}{l}18.9 \\
15.7\end{array}$ & $\begin{array}{r}7.4 \\
12.1\end{array}$ & $\begin{array}{l}21.9 \\
17.3\end{array}$ & $\begin{array}{l}0.82 \\
1.0\end{array}$ & $\begin{array}{l}0.94 \\
0.50\end{array}$ & $\begin{array}{l}46.1 \\
30.5\end{array}$ & 5. & $\begin{array}{l}32.4 \\
15.5\end{array}$ & $\begin{array}{l}10.5 \\
11.7\end{array}$ & $\begin{array}{l}492 \\
247\end{array}$ \\
\hline $9-3,90-98$ & 645 & 10 & 35 & 8 & 64 & 33 & 12 & 5 & & 90 & 41 & 2.5 & 105.2 & 0.3 & 3.6 & 3.7 & 20.1 & 9.9 & 13.6 & 0.8 & 0.8 & 34.0 & 4.2 & 21.3 & 9.8 & 431 \\
\hline
\end{tabular}


TABLE 4

Content of $\mathrm{C}_{\text {org }}$ and $\mathrm{CaCO}_{3}$ in Sediments and Sedimentary Rocks, in \% of Dry Weight (Wet Chemistry)

\begin{tabular}{|c|c|c|c|c|}
\hline $\begin{array}{c}\text { Sample } \\
\text { (Interval in } \mathrm{cm} \text { ) }\end{array}$ & $\begin{array}{l}\text { Depth } \\
\text { (m) }\end{array}$ & $\mathrm{CO}_{2}$ & $\mathrm{C}_{\text {org }}$ & $\mathrm{CaCO}_{3}$ \\
\hline \multicolumn{5}{|l|}{ Site 322} \\
\hline $1-2,26-30$ & 80 & & 0.03 & \\
\hline $1-3,70-76$ & 81 & 0.03 & 0.07 & 0.07 \\
\hline $2-2,113-116$ & 193 & 0.16 & 0.37 & 0.36 \\
\hline $3-1,18-20$ & 295 & & 0.08 & \\
\hline $4-1,65-67$ & 353 & 0.11 & 0.30 & 0.25 \\
\hline $4-2,57-60$ & 354 & 0.03 & 0.20 & 0.07 \\
\hline $5-1,81-85$ & 391 & 0.34 & 0.22 & 0.77 \\
\hline $6-1,30-33$ & 438 & 0.25 & 0.03 & 0.57 \\
\hline $9-2,143-146$ & 469 & 0.04 & 0.12 & 0.09 \\
\hline $10-1,101-105$ & 486 & 0.08 & 0.07 & 0.18 \\
\hline $10-2,43-46$ & 487 & & 0.04 & \\
\hline $11-1,42-46$ & 505 & 0.03 & 0.09 & 0.07 \\
\hline $11-2,45-49$ & 506 & 0.08 & 0.06 & 0.18 \\
\hline $11-3,24-32$ & 507 & 0.11 & 0.06 & 0.25 \\
\hline $11-4,17-24$ & 508 & 0.18 & 0.06 & 0.41 \\
\hline $11-5,36-40$ & 510 & 0.08 & 0.05 & 0.18 \\
\hline $11-6,109-115$ & 512 & 0.13 & 0.02 & 0.30 \\
\hline $13-2$ & 537 & 0.12 & 0.02 & 0.27 \\
\hline \multicolumn{5}{|l|}{ Site 323} \\
\hline $1-1,50-60$ & 76 & 0.04 & 0.07 & 0.09 \\
\hline $1-1,140-150$ & 77 & 0.26 & 0.07 & 0.59 \\
\hline $1-2,34-43$ & 79 & 0.36 & 0.07 & 0.82 \\
\hline $1-3,78-88$ & 80 & & 0.15 & \\
\hline $1-4,118-127$ & 81 & 0.06 & 0.14 & 0.14 \\
\hline $2-1,128-130$ & 162 & & 0.05 & \\
\hline $3-1,88-94$ & 257 & 0.06 & 0.40 & 0.14 \\
\hline $3-2,14-20$ & 258 & 0.22 & 0.13 & 0.50 \\
\hline $3-2,61-70$ & 258 & 0.10 & 0.06 & 0.23 \\
\hline $4-2$ & 315 & 0.40 & 0.05 & 0.91 \\
\hline $6-1,100-110$ & 343 & 0.09 & 0.23 & 0.21 \\
\hline $6-1,140-142$ & 343 & 0.03 & 0.05 & 0.07 \\
\hline $7-1,141-143$ & 362 & & 0.08 & \\
\hline $7-2,105-110$ & 363 & & 0.09 & \\
\hline $7-3,18-24$ & 364 & 0.06 & 0.08 & 0.14 \\
\hline $8-1,133-143$ & 409 & & 0.55 & \\
\hline $10-1,113-122$ & 504 & 0.04 & 0.06 & 0.09 \\
\hline $10-2,75-85$ & 505 & 0.08 & 0.14 & 0.18 \\
\hline $10-3,106-114$ & 507 & 0.05 & 0.10 & 0.11 \\
\hline $11-1,22-32$ & 551 & 0.20 & 0.23 & 0.45 \\
\hline $11-2,137-148$ & 553 & 0.18 & 0.32 & 0.41 \\
\hline $12-1,110-114$ & 599 & & 0.56 & \\
\hline $12-2,9-18$ & 599 & 0.29 & 0.12 & 0.66 \\
\hline $12-2,73-77$ & 600 & & 0.05 & \\
\hline $12-2,100-104$ & 600 & & 0.26 & \\
\hline $13-5,63-70$ & 623 & 0.53 & 0.11 & 1.20 \\
\hline $13-5,105-115$ & 624 & 0.08 & 0.06 & 0.18 \\
\hline $13-6,145-150$ & 625 & 0.50 & 0.04 & 1.14 \\
\hline $14-2,1-8$ & 637 & 0.08 & 0.03 & 0.18 \\
\hline $14-2,65-73$ & 638 & 0.26 & 0.05 & 0.59 \\
\hline $14-2,128-135$ & 638 & 0.07 & 0.04 & 0.16 \\
\hline $15-1,52-60$ & 656 & & & \\
\hline $15-2,91-100$ & 657 & 0.08 & 0.03 & 0.18 \\
\hline $15-3,29-36$ & 658 & 0.18 & & 0.41 \\
\hline $15-4,54-63$ & 660 & 0.14 & & 0.31 \\
\hline $15-5,89-98$ & 661 & 0.12 & 0.03 & 0.27 \\
\hline
\end{tabular}

TABLE 4 - Continued

\begin{tabular}{lcrcc}
\hline $\begin{array}{c}\text { Sample } \\
\text { (Interval in cm) }\end{array}$ & $\begin{array}{c}\text { Depth } \\
(\mathrm{m})\end{array}$ & $\mathrm{CO}_{2}$ & $\mathrm{C}_{\text {org }}$ & $\mathrm{CaCO}_{3}$ \\
\hline $15-6,19-26$ & 663 & 12.95 & 0.05 & 29.5 \\
$16-1,57-62$ & 665 & 12.74 & 0.05 & 28.8 \\
$16-3,32-37$ & 668 & 0.14 & 0.04 & 0.31 \\
$16-4,83-92$ & 669 & 0.06 & 0.05 & 0.14 \\
$18-2,80-86$ & 695 & & 0.08 & \\
$18-3,130-139$ & 697 & & 0.05 & \\
$18-4,115-124$ & 699 & 0.12 & 0.02 & 0.27 \\
$18-5,14-20$ & 700 & 0.03 & 0.03 & 0.07 \\
$18-5,65-73$ & 700 & 0.04 & 0.15 & 0.09
\end{tabular}

Site 324

$\begin{array}{lrlll}1-2,121-131 & 11 & & 0.06 & \\ 1-3,124-132 & 13 & 0.47 & 0.11 & 1.06 \\ 1-4,125-131 & 15 & & 0.19 & \\ 1-6,31-38 & 17 & 0.05 & 0.04 & 0.11 \\ 2-1,83-92 & 48 & & 0.22 & \\ 2-2,61-70 & 49 & & 0.40 & \\ 2-3,52-61 & 50 & 0.06 & 0.08 & 0.14 \\ 2-4,27-36 & 52 & 0.04 & 0.07 & 0.09 \\ 2-5,35-45 & 53 & 0.03 & 0.37 & 0.07 \\ 2-6,95-104 & 55 & 0.31 & 0.37 & 0.71 \\ 3-1,137-146 & 77 & 0.15 & 0.28 & 0.34 \\ 3-2,136-145 & 79 & 0.18 & 0.40 & 0.41 \\ 3-3,67-77 & 80 & 0.38 & 0.40 & 0.84 \\ 3-4,47-57 & 81 & 0.48 & 0.40 & 1.09 \\ 3-5,99-110 & 83 & 0.13 & 0.40 & 0.29 \\ 3-6,138-148 & 85 & & 0.08 & \\ 4-2,113-123 & 107 & 0.34 & 0.38 & 0.77 \\ 4-3,30-40 & 108 & 0.31 & 0.38 & 0.71 \\ 4-4,54-64 & 109 & 0.64 & 0.43 & 1.45 \\ 5-2,66-83 & 135 & 0.21 & 0.44 & 0.48 \\ 5-3,25-35 & 136 & 0.10 & 0.09 & 0.23 \\ 6-2,19-30 & 144 & 0.80 & 0.42 & 1.81 \\ 7-1,69-78 & 153 & 0.41 & 0.34 & 0.93 \\ 7-3,110-121 & 156 & 0.57 & 0.32 & 1.30 \\ 7-6,119-129 & 161 & 0.62 & 0.36 & 1.41 \\ 8-3,61-71 & 174 & 0.73 & 0.27 & 1.66\end{array}$

Site 325

\begin{tabular}{lrrrr}
$1-1,49-59$ & 34 & 0.20 & 0.09 & 0.45 \\
$1-1,133-144$ & 35 & 0.91 & 0.08 & 2.08 \\
$1-2,26-36$ & 36 & 0.14 & 0.05 & 0.31 \\
$1-4,81-92$ & 39 & 0.28 & 0.10 & 0.64 \\
$2-2,39-49$ & 168 & 0.14 & 0.15 & 0.31 \\
$3-2,11-24$ & 178 & 0.04 & 0.19 & 0.09 \\
$3-3,76-87$ & 180 & 0.08 & 0.17 & 0.18 \\
$3-4,26-36$ & 182 & 0.10 & 0.17 & 0.23 \\
$4-2,90$ & 293 & 0.64 & 0.05 & 1.46 \\
$4-3,20-26$ & 294 & 0.40 & 0.09 & 0.91 \\
$4-3,130$ & 295 & 0.30 & 0.08 & 0.68 \\
4, CC & 295 & 0.38 & 0.08 & 0.84 \\
5, CC & 407 & 0.04 & 0.14 & 0.09 \\
$6-1,145-150$ & 482 & & 0.31 & \\
$7-1,131-135$ & 520 & 0.04 & 0.15 & 0.09 \\
$7-2,138-142$ & 521 & 0.17 & 0.18 & 0.39 \\
$8-1,45-49$ & 613 & & 0.23 & \\
$8-2,119-128$ & 615 & 6.31 & 0.19 & 14.40 \\
$8-3,27-35$ & 616 & 0.21 & 0.08 & 0.48 \\
$9-3,90-98$ & 645 & & 0.15 & \\
$10-1,35-39$ & 709 & 0.13 & 0.15 & 0.29 \\
$10-3,99-102$ & 713 & 0.29 & 0.05 & 0.66 \\
\hline
\end{tabular}


TABLE 5

Content of $\mathrm{P}_{2} \mathrm{O}_{5}$ (in \% of Dry Weight) and Value of $\mathrm{C}_{\text {org }} / \mathrm{P}$ Ratio in Sediments and Sedimentary Rocks

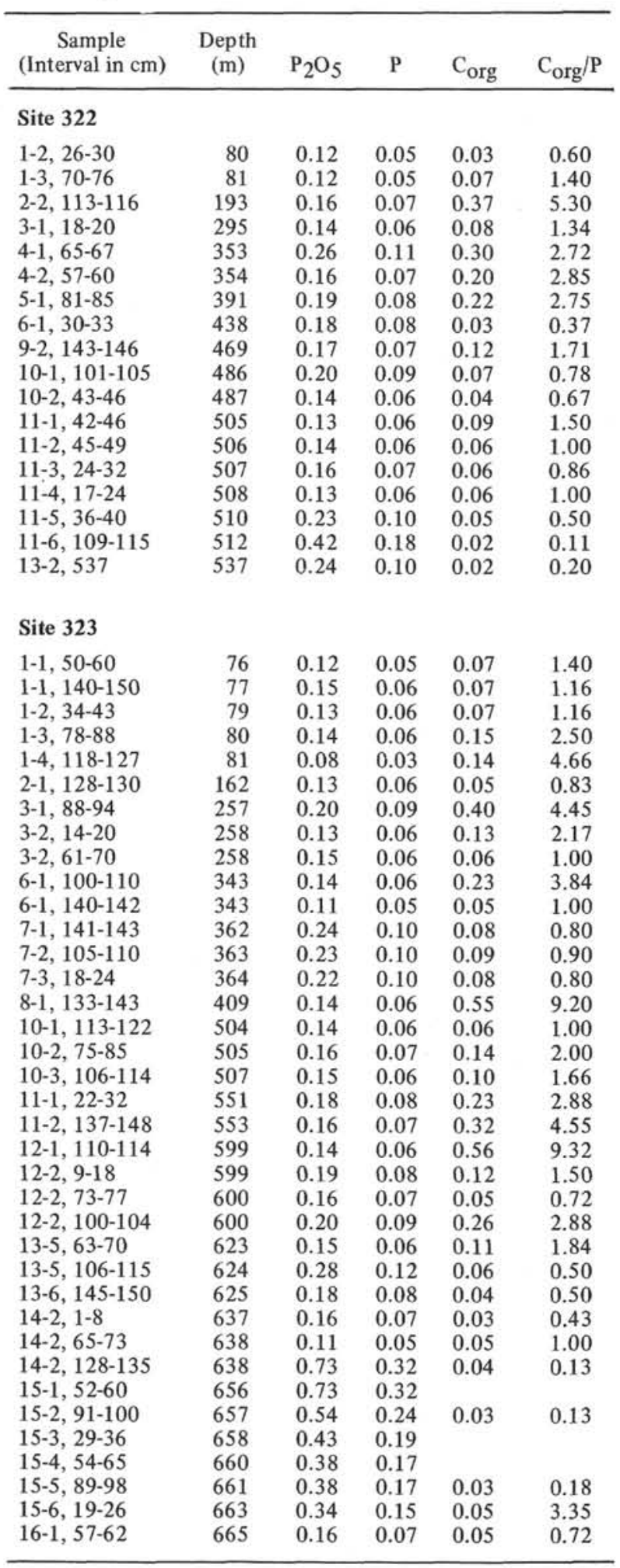

TABLE 5 - Continued

\begin{tabular}{lccccc}
\hline $\begin{array}{c}\text { Sample } \\
\text { (Interval in cm) }\end{array}$ & $\begin{array}{c}\text { Depth } \\
(\mathrm{m})\end{array}$ & $\mathrm{P}_{2} \mathrm{O}_{5}$ & $\mathrm{P}$ & $\mathrm{C}_{\text {org }}$ & $\mathrm{C}_{\text {org }} / \mathrm{P}$ \\
\hline $16-3,32-37$ & 668 & 0.33 & 0.14 & 0.04 & 0.29 \\
$16-4,83-92$ & 669 & 0.27 & 0.12 & 0.05 & 4.17 \\
$18-2,80-86$ & 695 & 0.19 & 0.08 & 0.08 & 1.00 \\
$18-3,130-139$ & 697 & 0.14 & 0.06 & 0.05 & 0.75 \\
$18-4,115-124$ & 699 & 0.20 & 0.09 & 0.02 & 0.22 \\
$18-5,14-20$ & 700 & 0.19 & 0.08 & 0.03 & 0.37 \\
$18-5,65-73$ & 700 & 0.30 & 0.13 & 0.15 & 1.15
\end{tabular}

Site 324

$\begin{array}{lrllll}1-2,126-131 & 11 & 0.18 & 0.08 & 0.06 & 0.75 \\ 1-3,124-132 & 13 & 0.20 & 0.09 & 0.11 & 1.22 \\ 1-4,125-131 & 15 & 0.19 & 0.08 & 0.19 & 2.39 \\ 1-6,31-38 & 17 & 0.22 & 0.10 & 0.04 & 0.40 \\ 2-1,83-92 & 48 & 0.22 & 0.10 & 0.22 & 2.20 \\ 2-2,61-70 & 49 & 0.32 & 0.14 & 0.40 & 2.85 \\ 2-3,52-61 & 50 & 0.21 & 0.09 & 0.08 & 0.89 \\ 2-4,27-36 & 52 & 0.19 & 0.08 & 0.07 & 0.88 \\ 2-5,35-45 & 53 & 0.19 & 0.08 & 0.37 & 4.63 \\ 2-6,95-104 & 55 & 0.21 & 0.09 & 0.37 & 4.12 \\ 3-1,137-146 & 77 & 0.20 & 0.09 & 0.28 & 3.12 \\ 3-2,136-145 & 79 & 0.18 & 0.08 & 0.40 & 5.00 \\ 3-3,67-77 & 80 & 0.20 & 0.09 & 0.40 & 4.45 \\ 3-4,45-57 & 81 & 0.22 & 0.10 & 0.40 & 4.00 \\ 3-5,99-110 & 83 & 0.19 & 0.08 & 0.40 & 5.00 \\ 3-6,138-148 & 85 & 0.16 & 0.07 & 0.08 & 1.14 \\ 4-2,113-123 & 107 & 0.21 & 0.09 & 0.38 & 4.21 \\ 4-3,30-40 & 108 & 0.20 & 0.09 & 0.38 & 4.21 \\ 4-4,54-64 & 109 & 1.28 & 0.57 & 0.43 & 0.75 \\ 5-2,66-83 & 135 & 0.21 & 0.09 & 0.44 & 4.90 \\ 5-3,25-35 & 136 & 0.24 & 0.10 & 0.09 & 0.90 \\ 6-2,19-30 & 144 & 0.19 & 0.08 & 0.42 & 5.25 \\ 7-1,69-78 & 153 & 0.20 & 0.09 & 0.34 & 3.80 \\ 7-3,110-120 & 156 & 0.20 & 0.09 & 0.32 & 3.55 \\ 7-6,119-129 & 161 & 0.19 & 0.08 & 0.36 & 4.50 \\ 8-3,61-71 & 174 & 0.25 & 0.11 & 0.27 & 2.46\end{array}$

Site 325

\begin{tabular}{lrllll}
$1-1,49-59$ & 34 & 0.21 & 0.09 & 0.09 & 1.00 \\
$1-1,133-144$ & 35 & 0.17 & 0.07 & 0.08 & 1.14 \\
$1-2,26-36$ & 36 & 0.17 & 0.07 & 0.05 & 0.72 \\
$1-4,81-92$ & 39 & 0.21 & 0.09 & 0.10 & 1.11 \\
$2-2,39-49$ & 168 & 0.21 & 0.09 & 0.15 & 1.66 \\
$3-2,11-24$ & 178 & 0.21 & 0.09 & 0.19 & 2.12 \\
$3-3,76-87$ & 180 & 0.19 & 0.08 & 0.17 & 2.13 \\
$3-4,26-36$ & 182 & 0.21 & 0.09 & 0.17 & 1.88 \\
$4-2,90$ & 293 & 0.19 & 0.08 & 0.05 & 0.63 \\
$4-2$, & 293 & 0.13 & 0.06 & & \\
$4-3,20-26$ & 294 & 0.17 & 0.07 & 0.09 & 1.29 \\
$4-3,130$ & 295 & 0.19 & 0.08 & 0.08 & 1.00 \\
4, CC & 295 & 0.19 & 0.08 & 0.08 & 1.00 \\
$5-1,55-57$ & 404 & 0.18 & 0.08 & & \\
5, CC & 407 & 0.17 & 0.07 & 0.14 & 2.00 \\
$6-1,145-150$ & 482 & 0.21 & 0.09 & 0.32 & 3.45 \\
$7-1,131-135$ & 520 & 0.14 & 0.06 & 0.15 & 2.50 \\
$7-2,138-142$ & 521 & 0.15 & 0.06 & 0.18 & 3.00 \\
$8-1,45-49$ & 613 & 0.17 & 0.07 & 0.23 & 3.29 \\
$8-2,119-128$ & 615 & 0.19 & 0.08 & 0.19 & 2.37 \\
$8-3,27-35$ & 616 & 0.14 & 0.06 & 0.08 & 1.33 \\
$9-3,90-98$ & 645 & 0.20 & 0.09 & 0.15 & 1.67 \\
$10-1,35-39$ & 709 & 0.16 & 0.07 & 0.15 & 2.15 \\
$10-2,26-30$ & 710 & 0.47 & 0.20 & & \\
$10-3,99-102$ & 713 & 0.12 & 0.05 & 0.05 & 1.00 \\
\hline
\end{tabular}


TABLE 6

Content of $\mathrm{SiO}_{2}$ (Amorph) in Sediments and Sedimentary Rocks (in \% of Dry Weight)

\begin{tabular}{|c|c|c|c|c|}
\hline $\begin{array}{c}\text { Sample } \\
\text { (Interval in } \mathrm{cm} \text { ) }\end{array}$ & $\begin{array}{l}\text { Depth } \\
\text { (m) }\end{array}$ & $\begin{array}{c}\mathrm{SiO}_{2} \\
\text { (Amorph) }\end{array}$ & $\begin{array}{l}\text { Total } \\
\mathrm{SiO}_{2}\end{array}$ & $\begin{array}{c}\mathrm{SiO}_{2}(\mathrm{Amorph}) / \\
\Sigma \mathrm{SiO}_{2}\end{array}$ \\
\hline \multicolumn{5}{|l|}{ Site 322} \\
\hline $1-2,26-30$ & 80 & 1.32 & 64 & 2.1 \\
\hline $1-3,70-76$ & 81 & 5.74 & 66 & 8.7 \\
\hline $2-2,113-116$ & 193 & 12.42 & & \\
\hline $3-1,18-20$ & 295 & 3.72 & 50 & 7.5 \\
\hline $4-1,65-67$ & 353 & 4.80 & 54 & 8.9 \\
\hline $4-2,57-60$ & 354 & 4.36 & 64 & 6.9 \\
\hline $5-1,81-85$ & 391 & 1.90 & 56 & 3.4 \\
\hline $6-1,30-33$ & 438 & 3.21 & 56 & 5.8 \\
\hline $9-2,143-146$ & 469 & 2.00 & 57 & 3.5 \\
\hline $10-1,101-105$ & 486 & 1.86 & 66 & 2.8 \\
\hline $10-2,43-46$ & 487 & 1.30 & 62 & 2.1 \\
\hline $11-1,42-46$ & 505 & 0.88 & & \\
\hline $11-2,45-49$ & 506 & 0.90 & 66 & 1.4 \\
\hline $11-3,24-32$ & 507 & 1.04 & 68 & 1.5 \\
\hline $11-4,17-24$ & 508 & 1.20 & 63 & 1.9 \\
\hline $11-5,36-40$ & 510 & 1.13 & & \\
\hline $11-6,109-115$ & 512 & 0.70 & 58 & 1.2 \\
\hline $13-2$ & 537 & 0.60 & 50 & 1.2 \\
\hline \multicolumn{5}{|l|}{ Site 323} \\
\hline $1-1,50-60$ & 76 & 1.70 & 56 & 3.1 \\
\hline $1-1,140-150$ & 77 & 4.60 & 57 & 8.1 \\
\hline $1-2,34-43$ & 79 & 7.45 & 56 & 1.3 \\
\hline $1-3,78-88$ & 80 & 12.00 & 52 & 23 \\
\hline $1-4,118-127$ & 81 & 12.80 & 54 & 25 \\
\hline $2-1,128-130$ & 162 & 6.55 & & \\
\hline $2-2,61-70$ & 162 & 3.22 & & \\
\hline $3-1,88-94$ & 257 & 4.49 & 55 & 8.1 \\
\hline $3-2,14-20$ & 258 & 2.39 & 58 & 4.1 \\
\hline $3-2,61-70$ & 258 & 4.76 & 56 & 8.5 \\
\hline $4-2$ & 315 & 1.32 & 55 & 2.4 \\
\hline $6-1,100-110$ & 343 & 10.67 & & \\
\hline $7-1,141-143$ & 362 & 4.55 & & \\
\hline $7-2,105-110$ & 363 & 5.20 & 52 & 10 \\
\hline $7-3,18-24$ & 364 & 5.23 & 58 & 9.1 \\
\hline $8-1,133-143$ & 409 & 10.62 & & \\
\hline $10-1,113-122$ & 504 & 2.04 & 64 & 3.2 \\
\hline $10-2,75-85$ & 505 & 4.48 & 60 & 7.5 \\
\hline $10-3,106-114$ & 507 & 2.85 & 60 & 4.8 \\
\hline $11-1,22-32$ & 551 & 4.80 & 55 & 8.7 \\
\hline $11-2,137-148$ & 553 & 5.12 & 51 & 10 \\
\hline $12-1,110-114$ & 599 & 1.90 & & \\
\hline $12-2,9-18$ & 599 & 1.50 & & \\
\hline $12-2,73-77$ & 600 & 1.00 & & \\
\hline $12-2,100-104$ & 600 & 0.73 & & \\
\hline $13-5,63-70$ & 623 & 4.53 & & \\
\hline $13-5,106-115$ & 624 & 1.70 & 47 & 3.6 \\
\hline $13-6,145-150$ & 625 & 1.35 & 51 & 2.6 \\
\hline $14-2,1-8$ & 637 & 1.90 & 52 & 3.6 \\
\hline $14-2,65-73$ & 638 & 0.77 & & \\
\hline $14-2,128-135$ & 638 & 1.40 & 48 & 2.9 \\
\hline $15-1,52-60$ & 656 & 1.20 & 45 & 2.7 \\
\hline $15-2,91-100$ & 657 & 0.83 & 50 & 1.7 \\
\hline $15-3,29-36$ & 658 & 1.16 & 47 & 2.5 \\
\hline $15-4,54-65$ & 660 & 1.23 & 45 & 2.7 \\
\hline $15-5,89-98$ & 661 & 0.84 & 46 & 1.8 \\
\hline $15-6,19-26$ & 663 & 0.80 & 33 & 2.4 \\
\hline
\end{tabular}

TABLE 6 - Continued

\begin{tabular}{lcccc}
\hline $\begin{array}{c}\text { Sample } \\
\text { (Interval in cm) }\end{array}$ & $\begin{array}{c}\text { Depth } \\
(\mathrm{m})\end{array}$ & $\begin{array}{c}\mathrm{SiO}_{2} \\
\text { amorph }\end{array}$ & $\mathrm{SiO}_{2}$ & $\begin{array}{c}\% \mathrm{SiO}_{2} \text { amorph } \\
\text { from } \mathrm{SiO}_{2}\end{array}$ \\
\hline $16-1,57-62$ & 665 & 1.22 & 34 & 3.6 \\
$16-3,32-37$ & 668 & 1.33 & 46 & 2.9 \\
$16-4,83-92$ & 669 & 2.30 & 61 & 3.8 \\
$18-2,80-86$ & 695 & 1.55 & 46 & 3.4 \\
$18-3,130-139$ & 697 & 2.30 & 45 & 5.1 \\
$18-4,115-124$ & 699 & 5.58 & 64 & 8.7 \\
$18-5,14-20$ & 700 & 2.86 & & \\
$18-5,65-73$ & 700 & 2.51 & 57 & 4.4
\end{tabular}

Site 324

$\begin{array}{lrlll}1-2,126-131 & 11 & 4.34 & 54 & 8.1 \\ 1-3,124-132 & 13 & 2.10 & 60 & 3.5 \\ 1-4,125-131 & 15 & 1.30 & 60 & 2.2 \\ 1-6,31-38 & 17 & 1.35 & 57 & 2.4 \\ 2-1,83-92 & 48 & 1.00 & 54 & 1.9 \\ 2-2,61-70 & 49 & 1.20 & 54 & 2.2 \\ 2-3,52-61 & 50 & 1.20 & 58 & 2.1 \\ 2-4,27-36 & 52 & 1.00 & 54 & 1.9 \\ 2-5,35-45 & 53 & 1.92 & 60 & 3.2 \\ 2-6,95-104 & 55 & 2.00 & 54 & 3.7 \\ 3-1,137-146 & 77 & 1.05 & 58 & 1.8 \\ 3-2,136-145 & 79 & 2.00 & 56 & 3.6 \\ 3-3,67-77 & 80 & 1.70 & 52 & 3.3 \\ 3-4,47-57 & 81 & 1.70 & 65 & 2.6 \\ 3-5,99-110 & 83 & 1.00 & 62 & 1.6 \\ 3-6,138-148 & 85 & 1.28 & 67 & 1.9 \\ 4-2,113-123 & 107 & 1.70 & 64 & 2.7 \\ 4-3,30-40 & 108 & 2.00 & 60 & 3.3 \\ 4-4,54-64 & 109 & 1.70 & 66 & 2.6 \\ 5-2,66-83 & 135 & 2.00 & 62 & 3.2 \\ 5-3,25-35 & 136 & 1.00 & 68 & 1.5 \\ 6-2,19-30 & 144 & 0.70 & 60 & 1.2 \\ 7-1,69-78 & 153 & 1.00 & 62 & 1.6 \\ 7-3,110-121 & 156 & 1.15 & 58 & 2.0 \\ 7-6,119-129 & 161 & 1.00 & 63 & 1.6 \\ 8-3,61-71 & 174 & 1.25 & & \end{array}$

Site 325

1-1, 49-59

1-1, 133-144

$1-2,26-36$

$1-4,81-92$

$2-2,39-49$

$3-2,11-24$

$3-3,76-87$

3-4, 26-36

$4-2,90$

4-3, $20-26$

$4-3,130$

$4, \mathrm{CC}$

5-1, 55-57

$5, \mathrm{CC}$

6-1, 145-150

$7-1,131-135$

$7-2,138-142$

$8-1,45-49$

8-2, 119-128

8-3, 24-35

9-3, 90-98

10-1, 35-39

34
35
36
39
168
178
180
182
293
294
295
295
405
407
482
520
521
613
615
616
645
709

2.00

2.70

3.20

1.87

11.40

10.30

9.60

10.00

1.45

1.65

1.49

1.50

5.17

7.64

13.47

5.74

5.80
11.51

11.51

9.88
8.70

1.80

1.80
1.44 
TABLE 7

Content of $\mathrm{CaO}$ and $\mathrm{MgO}$ (in \% of Dry Weight) and the Values of the $\mathrm{CaO} / \mathrm{MgO}$ Ratio in Sediments and Sedimentary Rocks

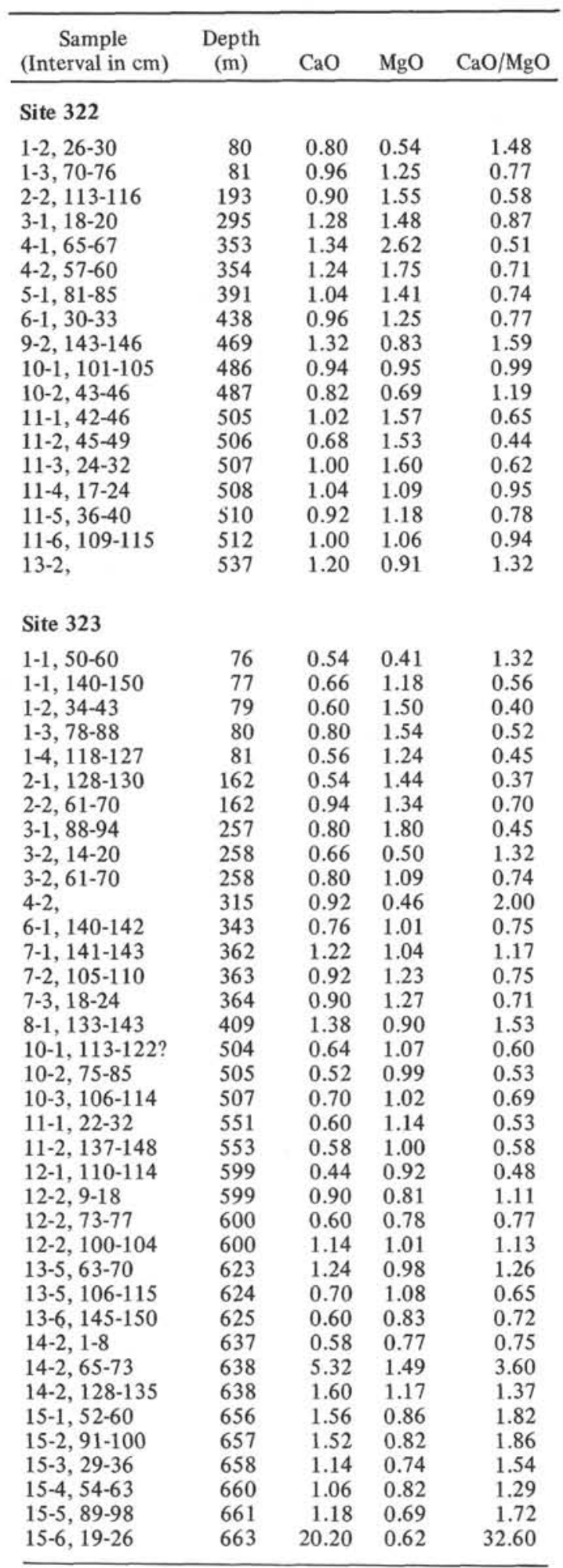

TABLE 7 - Continued

\begin{tabular}{lrrrc}
\hline $\begin{array}{c}\text { Sample } \\
\text { (Interval in cm) }\end{array}$ & $\begin{array}{c}\text { Depth } \\
(\mathrm{m})\end{array}$ & $\mathrm{CaO}$ & $\mathrm{MgO}$ & $\mathrm{CaO} / \mathrm{MgO}$ \\
\hline $16-1,57-62$ & 665 & 14.46 & 0.80 & 18.00 \\
$16-3,32-37$ & 668 & 1.20 & 0.69 & 1.74 \\
$16-4,83-92$ & 669 & 1.00 & 0.67 & 1.49 \\
$18-2,80-96$ & 695 & 0.98 & 0.61 & 1.61 \\
$18-3,130-139$ & 697 & 0.72 & 0.40 & 1.80 \\
$18-4,115-124$ & 699 & 0.80 & 0.81 & 0.99 \\
$18-5,14-20$ & 700 & 1.16 & 0.58 & 2.00 \\
$18-5,65-73$ & 700 & 1.00 & 1.12 & 0.90
\end{tabular}

Site 324

$\begin{array}{lrlll}1-2,126-131 & 11 & 0.70 & 1.52 & 0.46 \\ 1-3,124-132 & 13 & 0.80 & 1.44 & 0.56 \\ 1-4,125-131 & 15 & 0.80 & 0.93 & 0.86 \\ 1-6,31-38 & 17 & 0.65 & 1.23 & 0.53 \\ 2-1,83-92 & 48 & 0.64 & 1.24 & 0.52 \\ 2-2,61-70 & 49 & 0.68 & 1.04 & 0.65 \\ 2-3,52-61 & 50 & 0.96 & 1.28 & 0.75 \\ 2-4,27-36 & 52 & 0.72 & 0.83 & 0.87 \\ 2-5,35-45 & 53 & 0.82 & 1.41 & 0.58 \\ 2-6,95-104 & 55 & 0.80 & 1.48 & 0.54 \\ 3-1,137-146 & 77 & 0.72 & 1.15 & 0.63 \\ 3-2,136-145 & 79 & 1.24 & 1.23 & 1.01 \\ 3-3,67-77 & 80 & 0.90 & 1.14 & 0.79 \\ 3-4,45-47 & 81 & 0.90 & 1.34 & 0.67 \\ 3-5,99-110 & 83 & 0.50 & 1.37 & 0.36 \\ 3-6,138-148 & 85 & 0.60 & 1.03 & 0.58 \\ 4-2,113-123 & 107 & 0.52 & 1.21 & 0.43 \\ 4-3,30-40 & 108 & 0.86 & 1.04 & 0.83 \\ 4-4,54-64 & 109 & 2.52 & 1.24 & 2.04 \\ 5-2,66-83 & 135 & 0.74 & 1.08 & 0.69 \\ 5-3,25-35 & 136 & 0.60 & 1.36 & 0.44 \\ 6-2,19-30 & 144 & 1.28 & 1.14 & 1.12 \\ 7-1,69-78 & 153 & 1.00 & 1.09 & 0.92 \\ 7-3,110-121 & 156 & 0.56 & 1.28 & 0.44 \\ 7-6,119-129 & 161 & 1.00 & 1.48 & 0.68 \\ 8-3,61-71 & 174 & 1.31 & 1.16 & 1.13\end{array}$

Site 325

\begin{tabular}{lrrrl}
$1-1,49-59$ & 34 & 1.18 & 1.77 & 0.67 \\
$1-1,133-144$ & 35 & 2.46 & 1.74 & 1.41 \\
$1-2,26-36$ & 36 & 1.14 & 1.72 & 0.66 \\
$1-4,81-92$ & 39 & 0.90 & 1.51 & 0.59 \\
$2-2,39-49$ & 168 & 1.30 & 1.23 & 1.05 \\
$3-2,11-24$ & 178 & 1.00 & 1.37 & 0.73 \\
$3-3,76-87$ & 180 & 0.79 & 1.06 & 0.74 \\
$3-4,26-36$ & 182 & 0.84 & 1.07 & 0.79 \\
$4-2,90$ & 293 & 1.44 & 0.70 & 2.06 \\
$4-3,20-26$ & 294 & 1.40 & 0.52 & 2.70 \\
$4-3,130$ & 295 & 1.18 & 0.60 & 1.97 \\
4, CC & 295 & 1.52 & 0.82 & 1.85 \\
$5-1,55-57$ & 405 & 1.50 & 1.45 & 1.04 \\
5, CC & 407 & 1.30 & 1.33 & 0.98 \\
$6-1,145-150$ & 482 & 1.50 & 1.26 & 1.19 \\
$7-1,131-135$ & 520 & 1.42 & 0.99 & 1.44 \\
$7-2,138-142$ & 521 & 1.70 & 1.18 & 1.44 \\
$8-1,45-49$ & 613 & 1.32 & 1.24 & 1.06 \\
$8-2,119-128$ & 615 & 9.56 & 1.04 & 9.2 \\
$8-3,27-35$ & 616 & 1.54 & 1.24 & 1.24 \\
$9-3,90-98$ & 645 & 1.16 & 1.93 & 0.60 \\
$10-1,35-39$ & 709 & 1.12 & 1.82 & 0.62 \\
$10-2,26-30$ & 711 & 3.26 & 1.17 & 2.79 \\
$10-3,99-102$ & 713 & 1.04 & 2.41 & 0.43 \\
\hline
\end{tabular}


TABLE 8

Content of $\mathrm{Fe}_{2} \mathrm{O}_{3}, \mathrm{TiO}_{2}$, and $\mathrm{MnO}$ (in \% of Dry Weight) and Values of the $\mathrm{Fe}_{2} \mathrm{O}_{3} / \mathrm{MnO}$ Ratio in Sediments and Sedimentary Rocks

\begin{tabular}{cccccc}
\hline $\begin{array}{c}\text { Sample } \\
\text { (Interval in cm) }\end{array}$ & $\begin{array}{c}\text { Depth } \\
(\mathrm{m})\end{array}$ & $\mathrm{Fe}_{2} \mathrm{O}_{3}$ & $\mathrm{TiO}_{2}$ & $\mathrm{MnO}$ & $\mathrm{Fe}_{2} \mathrm{O}_{3} / \mathrm{MnO}$ \\
\hline
\end{tabular}

Site 322

$1-2,26-30$

$1-3,70-76$

2-2, 113-116

3-1, 18-20

4-1, 65-67

4-2, $57-60$

5-1, 81-85

6-1, 30-33

9-2, 143-146

$10-1,101-105$

10-2, 43-46

$11-1,42-46$

$11-2,45-49$

$11-3,24-32$

11-4, 17-24

$11-5,36-40$

11-6, 109-115

13-2,

80
81
193
295
353
354
391
438
469
486
487
505
506
507
508
510
512
537

$\begin{array}{rlll}3.84 & 0.48 & 0.06 & 63 \\ 5.60 & 0.58 & 0.08 & 70 \\ 6.51 & 0.56 & 0.10 & 65 \\ 6.90 & 0.72 & 0.10 & 69 \\ 8.07 & 0.72 & 0.12 & 67 \\ 7.54 & 0.70 & 0.11 & 69 \\ 7.50 & 0.85 & 0.30 & 25 \\ 7.63 & 0.67 & 0.87 & 8.8 \\ 5.88 & 0.64 & 0.10 & 59 \\ 4.93 & 0.56 & 0.08 & 61 \\ 4.61 & 0.56 & 0.07 & 66 \\ 4.93 & 0.64 & 0.07 & 71 \\ 5.07 & 0.62 & 0.08 & 63 \\ 5.05 & 0.64 & 0.08 & 63 \\ 4.70 & 0.64 & 0.09 & 52 \\ 8.64 & 0.68 & 1.08 & 8.0 \\ 9.54 & 0.64 & 1.13 & 8.4 \\ 12.72 & 1.60 & 0.17 & 75\end{array}$

Site 323

\begin{tabular}{|c|c|c|c|c|c|}
\hline $1-1,50-60$ & 76 & 2.69 & 0.40 & 0.09 & 30 \\
\hline $1-1,140-150$ & 77 & 5.48 & 0.60 & 0.06 & 15 \\
\hline $1-2,34-43$ & 79 & 7.13 & 0.62 & 0.09 & 79 \\
\hline $1-3,78-88$ & 80 & 6.68 & 0.60 & 0.09 & 74 \\
\hline $1-4,118-127$ & 81 & 6.48 & 0.60 & 0.08 & 81 \\
\hline $2-1,128-130$ & 162 & 8.27 & 0.60 & 0.12 & 69 \\
\hline $2-2,61-70$ & 163 & 7.13 & 0.58 & 0.06 & 119 \\
\hline $3-1,88-94$ & 257 & 6.09 & 0.62 & 0.09 & 68 \\
\hline $3-2,14-20$ & 258 & 3.43 & 0.44 & 0.05 & 69 \\
\hline $3-2,61-70$ & 258 & 7.02 & 0.64 & 0.04 & 17 \\
\hline $4-2$ & 315 & 3.99 & 0.40 & 0.09 & 45 \\
\hline $6-1,100-110$ & 343 & 5.60 & 0.52 & 0.12 & 47 \\
\hline $7-1,141-143$ & 362 & 8.93 & 0.50 & 0.16 & 56 \\
\hline $7-2,105-110$ & 363 & 7.98 & 0.62 & 0.13 & 61 \\
\hline $7-3,18-24$ & 364 & 7.18 & 0.62 & 0.14 & 51 \\
\hline $8-1,133-143$ & 409 & 5.05 & 0.51 & 0.06 & 84 \\
\hline $10-1,113-122$ & 504 & 6.92 & 0.64 & 0.07 & 99 \\
\hline $10-2,75-85$ & 505 & 7.12 & 0.70 & 0.07 & 102 \\
\hline $10-3,106-114$ & 507 & 6.96 & 0.64 & 0.06 & 105 \\
\hline $11-1,22-32$ & 551 & 6.12 & 0.64 & 0.11 & 56 \\
\hline $11-2,137-148$ & 553 & 5.56 & 0.64 & 0.10 & 56 \\
\hline $12-1,110-114$ & 599 & 5.99 & 0.66 & 0.06 & 100 \\
\hline $12-2,9-18$ & 599 & 5.70 & 0.67 & 0.06 & 95 \\
\hline $12-2,73-77$ & 600 & 6.12 & 0.64 & 0.14 & 44 \\
\hline $12-2,100-104$ & 600 & 6.00 & 0.64 & 0.07 & 86 \\
\hline $13-5,63-70$ & 623 & 7.22 & 0.61 & 0.33 & 22 \\
\hline $13-5,106-115$ & 624 & 7.18 & 0.72 & 0.21 & 34 \\
\hline $13-6,145-150$ & 625 & 5.64 & 0.74 & 0.12 & 47 \\
\hline $14-2,1-8$ & 637 & 5.75 & 0.60 & 0.12 & 48 \\
\hline $14-2,65-73$ & 638 & 4.80 & 0.66 & 0.16 & 30 \\
\hline $14-2,128-135$ & 638 & 10.51 & 0.74 & 1.92 & 55 \\
\hline $15-1,52-60$ & 656 & 12.17 & 0.74 & 2.56 & 4.7 \\
\hline $15-2,91-100$ & 657 & 10.77 & 0.76 & 1.56 & 6.9 \\
\hline $15-3,29-36$ & 658 & 10.93 & 0.76 & 1.55 & 7.1 \\
\hline $15-4,54-63$ & 660 & 11.03 & 0.78 & 1.55 & 7.1 \\
\hline $15-5,89-98$ & 661 & 10.67 & 0.64 & 1.87 & 5.7 \\
\hline $15-6,19-26$ & 663 & 7.71 & 0.42 & 1.33 & 5.8 \\
\hline $16-1,57-62$ & 665 & 6.46 & 0.58 & 2.41 & 2.7 \\
\hline
\end{tabular}

TABLE 8 - Continued

\begin{tabular}{lcrccc}
\hline $\begin{array}{c}\text { Sample } \\
\text { (Interval in cm) }\end{array}$ & $\begin{array}{c}\text { Depth } \\
(\mathrm{m})\end{array}$ & $\mathrm{Fe}_{2} \mathrm{O}_{3}$ & $\mathrm{TiO}_{2}$ & $\mathrm{MnO}$ & $\mathrm{Fe}_{2} \mathrm{O}_{3} / \mathrm{MnO}$ \\
\hline $16-3,32-37$ & 668 & 9.47 & 0.66 & 0.80 & 11.9 \\
$16-4,83-92$ & 669 & 10.00 & 0.68 & 0.57 & 16.7 \\
$18-2,80-86$ & 695 & 7.85 & 0.74 & 0.11 & 71 \\
$18-3,130-139$ & 697 & 7.47 & 0.60 & 0.09 & 83 \\
$18-4,115-124$ & 699 & 7.87 & 0.56 & 1.12 & 7.1 \\
$18-5,14-20$ & 700 & 7.94 & 0.51 & 0.27 & 29 \\
$18-5,65-73$ & 700 & 9.71 & 0.56 & 1.68 & 5.8
\end{tabular}

Site 324

$\begin{array}{lrrrrr}1-2,126-131 & 11 & 6.04 & 0.64 & 0.11 & 55 \\ 1-3,124-132 & 13 & 6.07 & 0.68 & 0.20 & 30 \\ 1-4,125-131 & 15 & 5.82 & 0.67 & 0.10 & 58 \\ 1-6,31-38 & 17 & 5.34 & 0.63 & 0.17 & 31 \\ 2-1,83-92 & 48 & 6.55 & 0.83 & 0.07 & 93 \\ 2-2,61-70 & 49 & 7.00 & 0.80 & 0.08 & 88 \\ 2-3,52-61 & 50 & 6.42 & 0.72 & 0.07 & 92 \\ 2-4,27-36 & 52 & 6.61 & 0.67 & 0.06 & 110 \\ 2-5,35-45 & 53 & 6.04 & 0.75 & 0.07 & 86 \\ 2-6,95-104 & 55 & 6.07 & 0.69 & 0.11 & 55 \\ 3-1,137-146 & 77 & 6.13 & 0.78 & 0.08 & 77 \\ 3-2,136-145 & 79 & 6.36 & 0.72 & 0.10 & 64 \\ 3-3,67-77 & 80 & 6.10 & 0.72 & 0.11 & 55 \\ 3-4,47-57 & 81 & 6.58 & 0.77 & 0.10 & 66 \\ 3-5,99-110 & 83 & 6.61 & 0.75 & 0.09 & 74 \\ 3-6,138-148 & 85 & 6.84 & 0.64 & 0.06 & 114 \\ 4-2,113-123 & 107 & 6.30 & 0.78 & 0.11 & 57 \\ 4-3,30-40 & 108 & 6.40 & 0.71 & 0.11 & 58 \\ 4-4,54-64 & 109 & 5.78 & 0.72 & 0.16 & 36 \\ 5-2,66-83 & 135 & 5.98 & 0.70 & 0.08 & 75 \\ 5-3,25-35 & 136 & 6.90 & 0.80 & 0.08 & 86 \\ 6-2,19-30 & 144 & 6.36 & 0.77 & 0.10 & 64 \\ 7-1,69-78 & 153 & 6.33 & 0.72 & 0.10 & 63 \\ 7-3,110-120 & 156 & 6.10 & 0.72 & 0.10 & 61 \\ 7-6,119-129 & 161 & 6.52 & 0.80 & 0.12 & 54 \\ 8-3,61-71 & 174 & 6.13 & 0.74 & 0.08 & 77\end{array}$

Site 325

\begin{tabular}{lrrrrr}
$1-1,49-59$ & 34 & 7.71 & 0.80 & 0.13 & 59 \\
$1-1,133-144$ & 35 & 7.29 & 0.80 & 0.12 & 61 \\
$1-2,26-36$ & 36 & 7.25 & 0.80 & 0.11 & 66 \\
$1-4,81-92$ & 39 & 6.52 & 0.76 & 0.09 & 73 \\
$2-2,39-49$ & 168 & 6.01 & 0.68 & 0.11 & 55 \\
$3-2,11-24$ & 178 & 6.68 & 0.74 & 0.09 & 74 \\
$3-3,76-87$ & 180 & 5.72 & 0.66 & 0.09 & 63 \\
$3-4,26-36$ & 182 & 5.72 & 0.64 & 0.09 & 63 \\
$4-2,90$ & 293 & 6.14 & 0.72 & 0.09 & 68 \\
$4-3,20-26$ & 294 & 6.52 & 0.74 & 0.11 & 59 \\
$4-3,130$ & 295 & 6.54 & 0.76 & 0.12 & 55 \\
4, CC & 295 & 6.68 & 0.74 & 0.13 & 51 \\
$5-1,55-57$ & 405 & 7.21 & 0.78 & 0.10 & 72 \\
5, CC & 407 & 7.52 & 0.64 & 0.07 & 108 \\
$6-1,145-150$ & 482 & 7.35 & 0.70 & 0.08 & 92 \\
$7-1,131-135$ & 520 & 6.94 & 0.56 & 0.07 & 99 \\
$7-2,138-142$ & 521 & 7.13 & 0.64 & 0.08 & 89 \\
$8-1,45-49$ & 613 & 6.83 & 0.68 & 0.10 & 68 \\
$8-2,119-128$ & 615 & 6.76 & 0.64 & 0.12 & 56 \\
$8-3,27-35$ & 616 & 7.34 & 0.70 & 0.09 & 81 \\
$9-3,90-98$ & 645 & 7.42 & 0.76 & 0.11 & 67 \\
$10-1,35-39$ & 709 & 8.31 & 0.72 & 0.10 & 83 \\
$10-2,26-30$ & 711 & 6.98 & 0.64 & 0.10 & 70 \\
$10-3,99-102$ & 713 & 8.25 & 0.64 & 0.10 & 83 \\
\hline
\end{tabular}


TABLE 9

Content of $\mathrm{C}_{\text {org }}$ and $\mathrm{CaCO}_{3}$ in the Sediments and Sedimentary Rocks (in \% of Dry Weight)

\begin{tabular}{ccccc}
\hline $\begin{array}{c}\text { Sample } \\
\text { (Interval in cm) }\end{array}$ & $\begin{array}{c}\text { Depth } \\
(\mathrm{m})\end{array}$ & $\mathrm{C}_{\text {org }}$ & $\mathrm{C}_{\text {carb }}$ & $\mathrm{CaCO}_{3}$ \\
\hline
\end{tabular}

Site 322

$1-2,26-30$

$1-3,70-76$

3-1, $18-20$

4-1, 65-67

4-2, $57-60$

$5-1,81-85$

6-1, 30-33

9-2, 143-146

$10-1,101-105$

$10-2,43-46$

$11-2,45-49$

$11-3,24-32$

11-4, 17-24

$11-6,109-115$

13-2,

$\begin{array}{rr}80 & 0.04 \\ 81 & 0.06 \\ 295 & 0.06 \\ 353 & 0.25 \\ 354 & 0.16 \\ 391 & 0.25 \\ 438 & 0.06 \\ 469 & 0.07 \\ 486 & 0.03 \\ 487 & 0.04 \\ 506 & 0.03 \\ 507 & 0.03 \\ 508 & 0.08 \\ 512 & 0.06 \\ 537 & 0.02\end{array}$

$0 . \overline{05}$

0.07

0.06

0.04

0.06

$0 . \overline{05}$

0.05

0.01

$0 . \overline{5}$

0.01

$0 . \overline{0}$

$0.4 \overline{16}$

0.583

0.50

0.334

0.50

0.416

0.416

0.0835

$0.4 \overline{16}$

0.0835

0.0835

Site 323

1-1, 50-6

$1-1,140-150$

1-2, 34-43

1-3, 78-83

$1-4,118-127$

3-1, 88-94

3-2, 14-20

3-2, 61-70

4-2,

7-2, 105-110

7-3, 18-24

10-1, 113-122

10-2, 75-85

10-3, 106-114

11-1, 22-32

11-2, 137-148

13-5, 106-115

13-6, 145-150

14-2, $1-8$

14-2, 128-135

15-1, 52-60

15-2, 91-100

15-3, 29-36

15-4, 54-63

15-5, 89-98

15-6, 19-26

16-1, 57-62

16-3, 32-37

16-4, 83-92

$18-2,80-86$

$18-3,130-139$

$18-4,115-124$

$18-5,65-73$

Site 324

1-2, 126-131

1-3, 124-132

$1-4,125-131$

1-6, 31-38

2-1, 83-92

2-2, 61-70

2-3, 52-61

2-4, 27-36

$2-5,35-45$

2-6, 95-104

3-1, 137-146

3-2, 136-145

3-3, 67-77
TABLE 9 - Continued

\begin{tabular}{|c|c|c|c|c|}
\hline $\begin{array}{c}\text { Sample } \\
\text { (Interval in } \mathrm{cm} \text { ) }\end{array}$ & $\begin{array}{l}\text { Depth } \\
\text { (m) }\end{array}$ & $\mathrm{C}_{\text {org }}$ & $\mathrm{C}_{\text {carb }}$ & $\mathrm{CaCO}_{3}$ \\
\hline \multicolumn{5}{|c|}{ Site 324 - Continued } \\
\hline $\begin{array}{l}3-4,47-57 \\
3-5,99-110 \\
3-6,138-148 \\
4-2,113-123 \\
4-3,30-40 \\
4-4,54-64 \\
5-2,66-83 \\
5-3,25-35 \\
6-2,19-30 \\
7-1,69-78 \\
7-3,110-121 \\
7-6,119-129 \\
8-3,61-71\end{array}$ & $\begin{array}{r}81 \\
83 \\
85 \\
107 \\
108 \\
109 \\
135 \\
136 \\
144 \\
153 \\
156 \\
161 \\
174\end{array}$ & $\begin{array}{l}0.51 \\
0.45 \\
0.17 \\
0.43 \\
0.41 \\
0.44 \\
0.43 \\
0.34 \\
0.39 \\
0.43 \\
0.44 \\
0.41 \\
0.35\end{array}$ & $\begin{array}{r}0.01 \\
0.02 \\
- \\
- \\
0.03 \\
0.07 \\
0.01 \\
0.01 \\
0.06 \\
0.06 \\
0.03 \\
0.08 \\
0.12\end{array}$ & 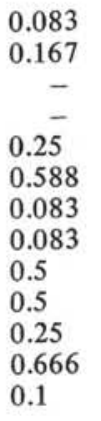 \\
\hline \multicolumn{5}{|l|}{ Site 325} \\
\hline $\begin{array}{l}1-1,49-59 \\
1-1,133-144 \\
1-2,26-36 \\
1-4,81-92 \\
2-2,39-49 \\
3-2,11-24 \\
3-3,76-87 \\
3-4,26-36 \\
4-2,90 \\
4-3,20-26 \\
4-3,130 \\
4, \text { CC } \\
5-1,55-57 \\
8-1,45-49 \\
8-2,119-128 \\
8-3,27-35 \\
9-3,90-98\end{array}$ & $\begin{array}{r}34 \\
35 \\
36 \\
39 \\
168 \\
178 \\
180 \\
182 \\
293 \\
294 \\
295 \\
295 \\
405 \\
613 \\
615 \\
616 \\
645\end{array}$ & $\begin{array}{l}0.10 \\
0.34 \\
0.11 \\
0.09 \\
0.21 \\
0.19 \\
0.13 \\
0.13 \\
0.11 \\
0.10 \\
0.07 \\
0.12 \\
0.15 \\
0.14 \\
0.88 \\
0.12 \\
0.11\end{array}$ & $\begin{array}{l}0.07 \\
\overline{-} \\
0.04 \\
0.05 \\
0.06 \\
0.06 \\
0.09 \\
0.07 \\
0.08 \\
0.08 \\
0.07 \\
0.06 \\
0.06 \\
0.04 \\
0.21 \\
0.07 \\
0.04\end{array}$ & $\begin{array}{l}0.583 \\
\quad- \\
0.333 \\
0.416 \\
0.5 \\
0.5 \\
0.75 \\
0.583 \\
0.666 \\
0.666 \\
0.583 \\
0.5 \\
0.5 \\
0.333 \\
0.175 \\
0.583 \\
0.333\end{array}$ \\
\hline
\end{tabular}

Thus in the cores of Site 323, two processes occur toward the end of the Paleogene. The highest concentrations of $\mathrm{Fe}, \mathrm{Mn}, \mathrm{Ni}, \mathrm{Co}, \mathrm{Cu}$, and rare earths were found in the uppermost horizons of the Paleogene sediments. At the boundary between the Paleogene and Neogene time an unusual change in the depositional environment occurred within 1 meter of sediment and the concentrations of all elements drastically decreased, whereas the concentrations of $\mathrm{Cr}$ and alkaline metals increased. The concentrations of trace elements were minimal in Neogene sediments from all Leg 35 sites. The range of variation in concentrations corresponds to that found in present-day iceberg deposits containing sediments of various textural composition (pelitic oozes to sands). The fluctuations in the trace element concentrations in the Neogene and Pleistocene sediments are apparently due to changes in intensity of terrigenous load which is related to periodic climatic changes. Evidence of such climatic changes was noted by Angino (1966) in the sediments of the Pacific and Atlantic sectors of the Antarctic region.

\section{REFERENCES}

Angino E.E., 1966. Geochemistry of Antarctic pelagic sediments: Geochim. Cosmochim. Acta, v. 30, p. 939-962.

El-Wakeel S.K. and Riley P., 1961. Chemical and mineralogical studies of deep-sea sediments: Geochim. Cosmochim. Acta, v. 25, p. 110-146. 
TABLE 10

Content of $\mathrm{CaO}$ and $\mathrm{MgO}$ in Pelagic Sediments and in Other Widely Distributed Rocks ${ }^{a}$

\begin{tabular}{|c|c|c|c|c|c|c|c|c|c|}
\hline \multirow{2}{*}{$\begin{array}{l}\text { Geologic } \\
\text { Formations }\end{array}$} & \multicolumn{3}{|c|}{ Carbonates } & \multicolumn{3}{|c|}{$\begin{array}{l}\text { Noncarbonates (converted } \\
\text { to noncalcareous oxides) }\end{array}$} & \multicolumn{3}{|c|}{ Total Content } \\
\hline & $\mathrm{CaO}$ & $\mathrm{Mg}$ & $\mathrm{CaO} / \mathrm{MgO}$ & $\mathrm{CaO}$ & $\mathrm{MgO}$ & $\mathrm{CaO} / \mathrm{MgO}$ & $\mathrm{CaO}$ & $\mathrm{MgO}$ & $\mathrm{CaO} / \mathrm{MgO}$ \\
\hline \multicolumn{10}{|l|}{$\begin{array}{l}\text { Oceanic } \\
\text { sediments }\end{array}$} \\
\hline $\begin{array}{l}\text { Carbonate } \\
\text { sediments }\end{array}$ & 28.05 & 1.03 & 27.2 & 1.04 & 3.05 & 0.34 & 28.35 & 2.35 & 12.2 \\
\hline $\begin{array}{l}\text { Siliceous } \\
\text { sediments }\end{array}$ & 0.61 & 0.49 & 1.2 & 0.83 & 2.17 & 0.38 & 1.36 & 2.44 & 0.56 \\
\hline $\begin{array}{l}\text { Pelitic } \\
\quad \text { oozes }\end{array}$ & 0.44 & 0.40 & 1.1 & 1.01 & 3.73 & 0.27 & 1.37 & 3.82 & 0.36 \\
\hline $\begin{array}{l}\text { Average } \\
\text { of ocean }\end{array}$ & 13.95 & 0.72 & 19.4 & 1.00 & 3.19 & 0.31 & 14.55 & 2.90 & 5.0 \\
\hline $\begin{array}{l}\text { Ultrabasic } \\
\text { rocks }\end{array}$ & - & - & - & - & - & - & 1.0 & 43.1 & 0.02 \\
\hline $\begin{array}{l}\text { Basic } \\
\text { rocks }\end{array}$ & - & - & - & - & - & - & 9.4 & 7.5 & 1.3 \\
\hline $\begin{array}{l}\text { Intermediate } \\
\text { rocks }\end{array}$ & - & - & - & - & - & - & 6.5 & 3.6 & 1.8 \\
\hline $\begin{array}{l}\text { Acid } \\
\text { rocks }\end{array}$ & - & - & - & - & - & - & 2.2 & 0.9 & 2.4 \\
\hline $\begin{array}{l}\text { Sedimentary } \\
\text { rocks }\end{array}$ & - & - & - & - & - & - & 3.5 & 2.2 & 1.6 \\
\hline Soil & - & - & - & - & - & - & 2.9 & 1.0 & 1.9 \\
\hline
\end{tabular}

aAccording to Wakeel and Riley, 1961; Vinogradov, 1962; Malyuga, 1963,

Malyuga, D.P., 1963. Biochemical method of the exploration of ore deposits: Publication of the Academy of Sciences USSR, Moscow.
Vinogradov, A.P., 1962. Average content of chemical elements in the main types of volcanic rocks of the earth's crust: Geochemistry, No. 7. 


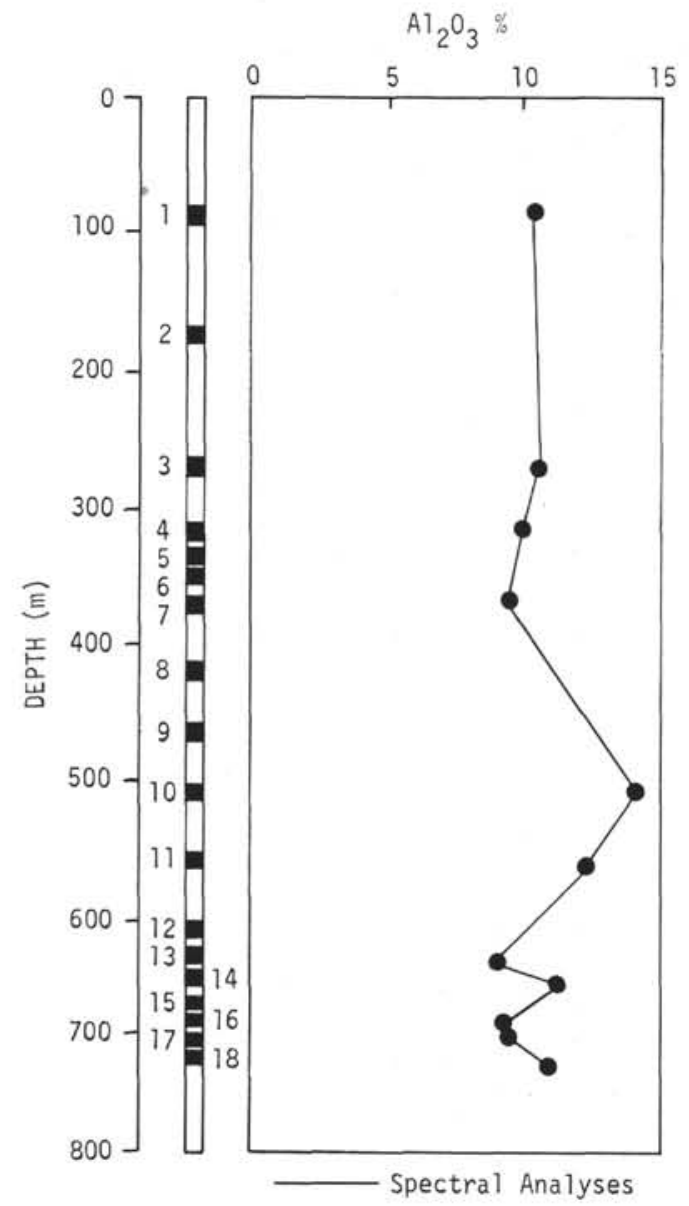

$\mathrm{SiO}_{2} \%$

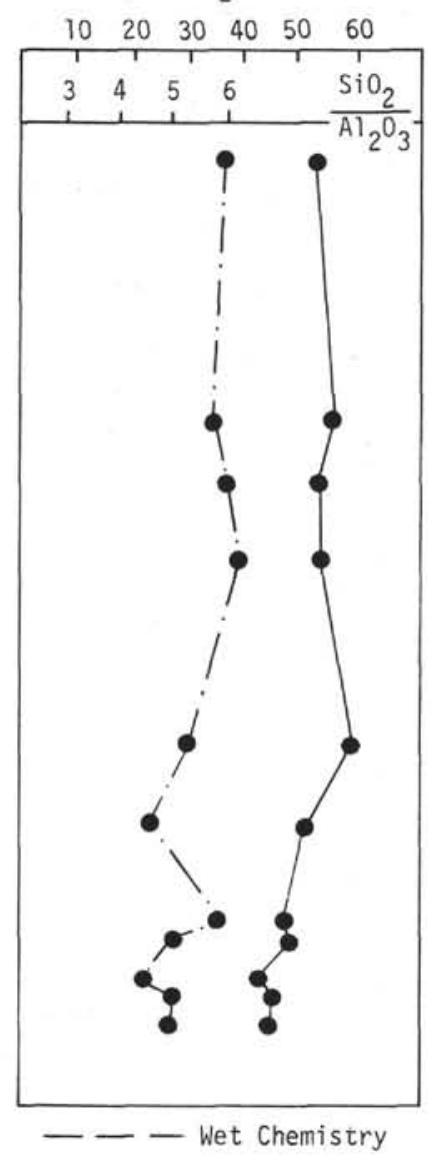

$\mathrm{TiO}_{2} \%$

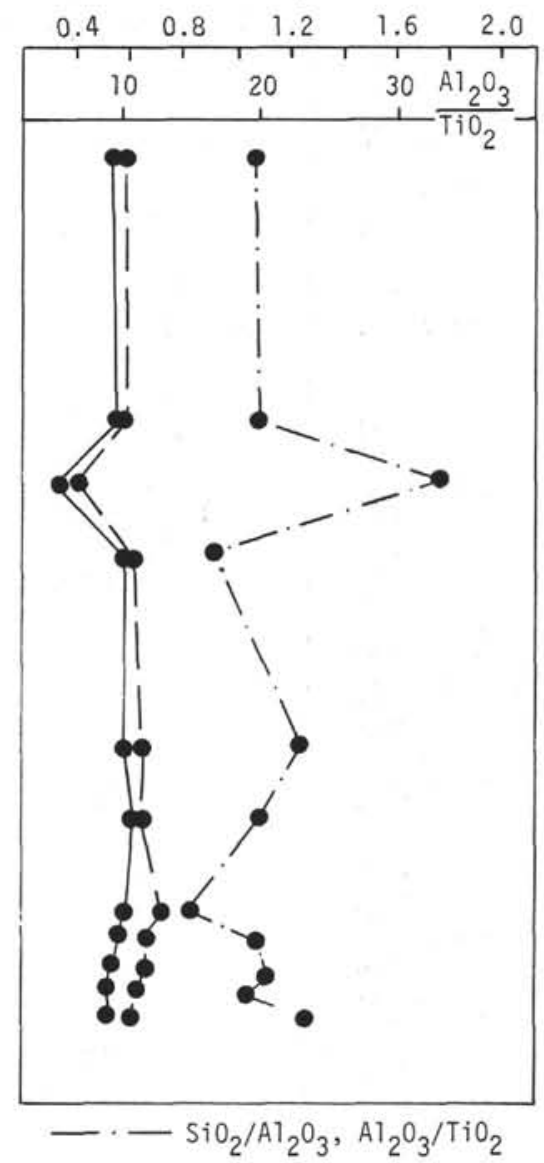

Figure 1. Analyses of $\mathrm{Al}_{2} \mathrm{O}_{3}, \mathrm{SiO}_{2}$, and $\mathrm{TiO}_{2}$ for Site 323. Values are the average for each core and are given in percent. 


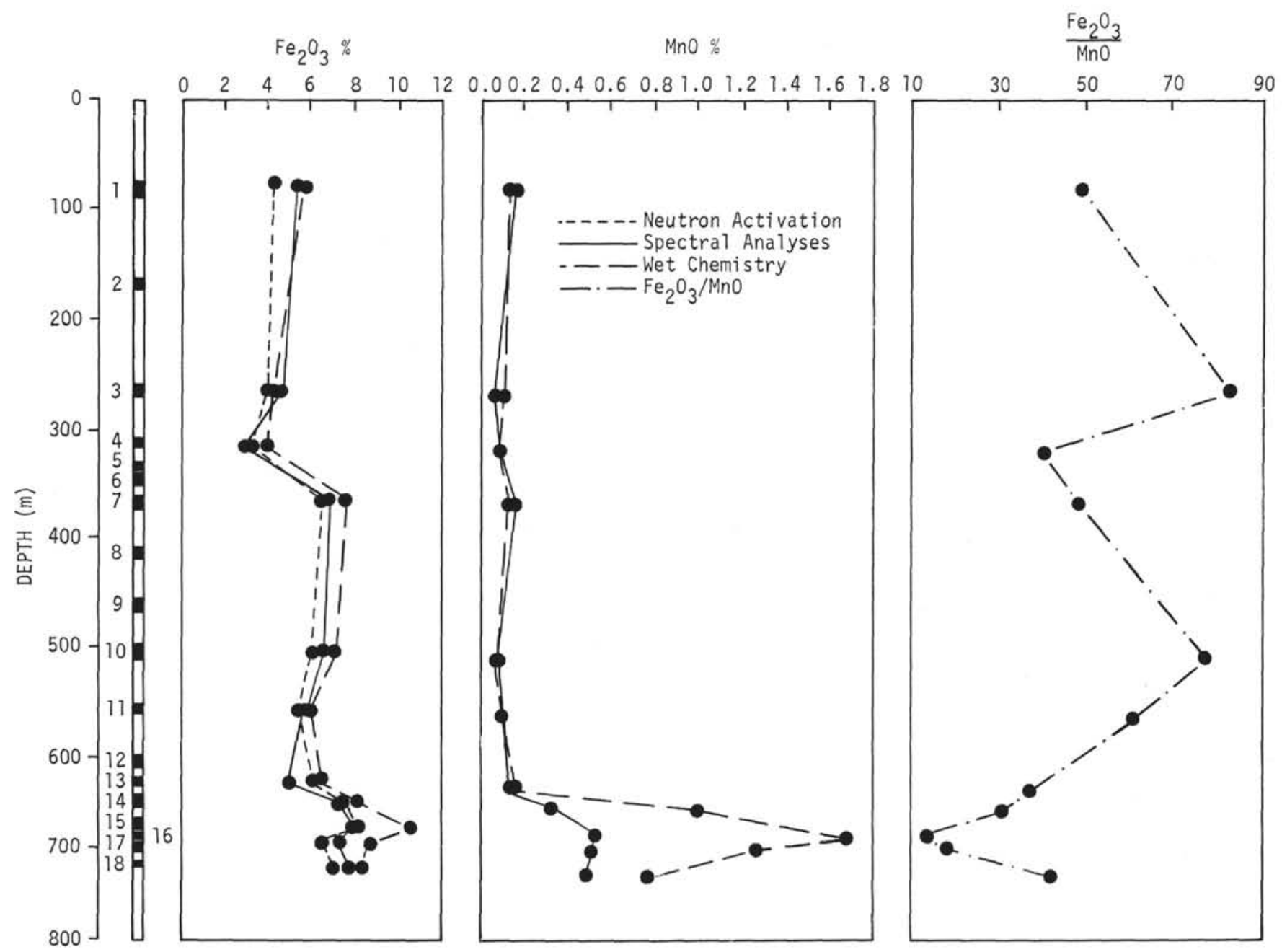

Figure 2. Analyses of $\mathrm{Fe}_{2} \mathrm{O}_{3}$ and $\mathrm{MnO}$ for Site 323. Values are the average for each core and are given in percent. 


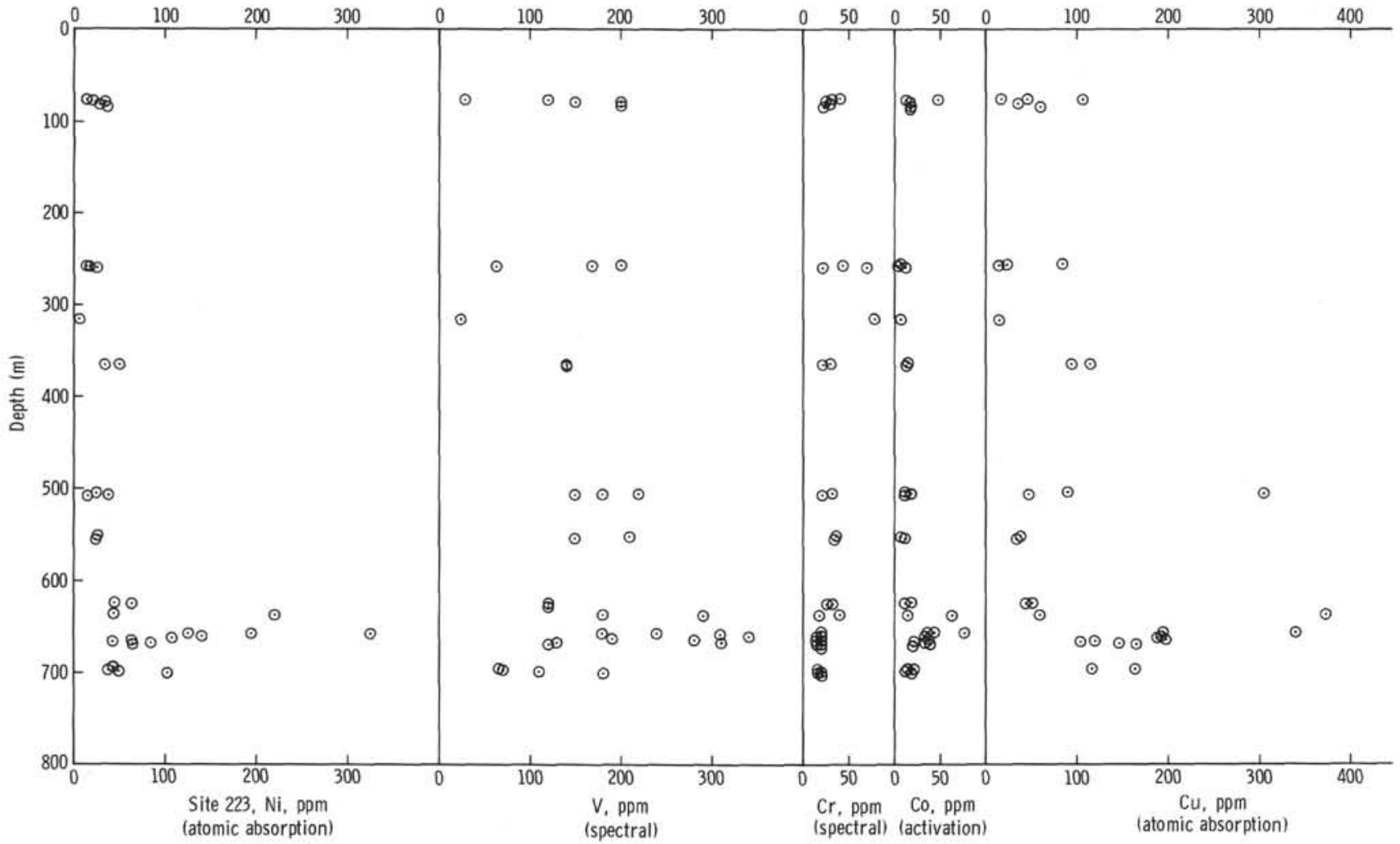

Figure 3. Analyses of selected trace elements, Fe, and Mn for Site 323. Values are plotted from Tables 1 and 3. 
Y. A. BOGDANOV ET AL.

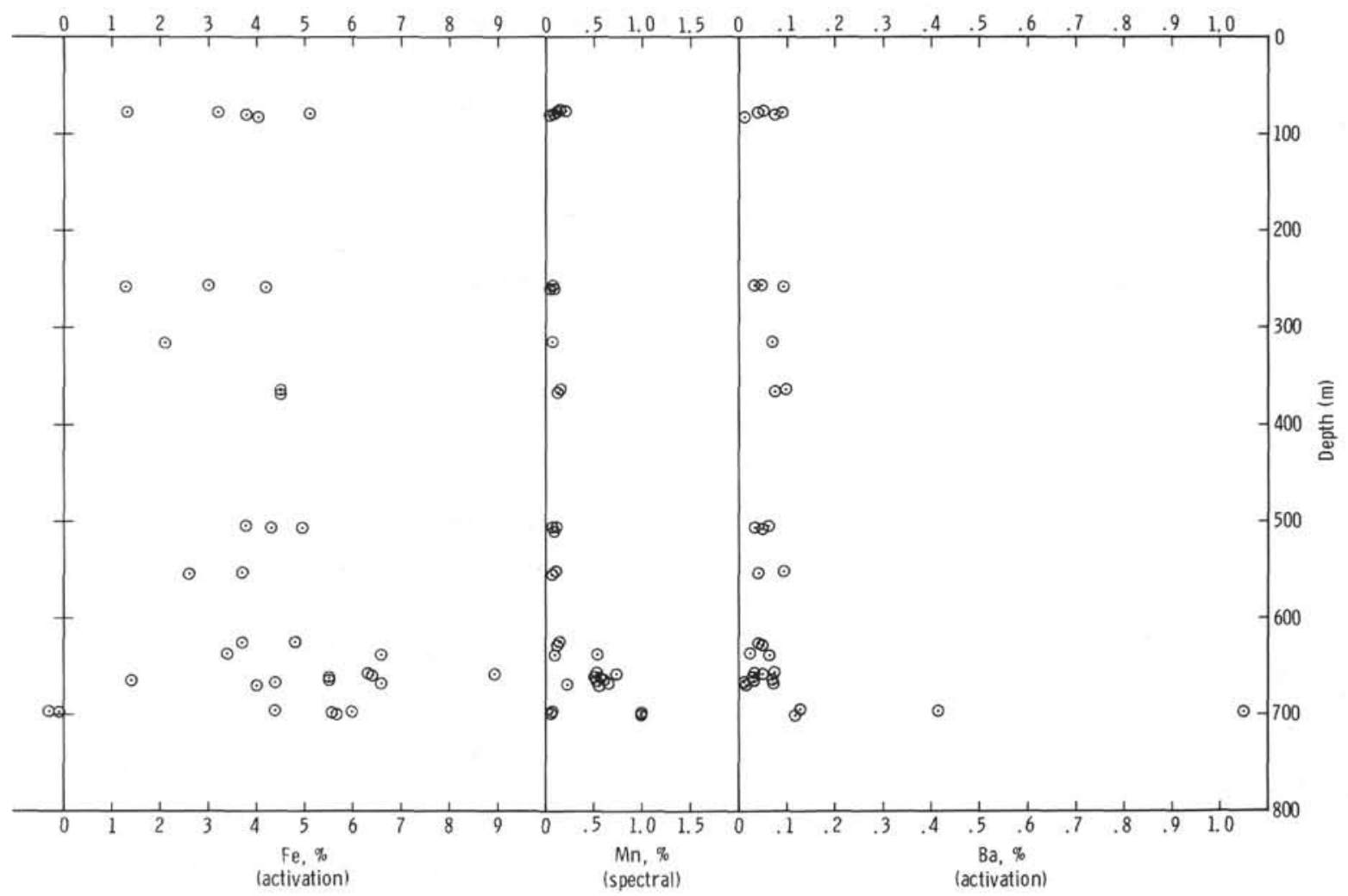

Figure 3. (Continued). 